\title{
Geometric intersection number and analogues of the curve complex for free groups
}

\author{
ILYA KAPOVICH \\ MARTIN LUSTIG
}

For the free group $F_{N}$ of finite rank $N \geq 2$ we construct a canonical Bonahon-type, continuous and $\operatorname{Out}\left(F_{N}\right)$-invariant geometric intersection form

$$
\langle,\rangle: \overline{\operatorname{cv}}\left(F_{N}\right) \times \operatorname{Curr}\left(F_{N}\right) \rightarrow \mathbb{R}_{\geq 0} .
$$

Here $\overline{\operatorname{cv}}\left(F_{N}\right)$ is the closure of unprojectivized Culler-Vogtmann Outer space $\operatorname{cv}\left(F_{N}\right)$ in the equivariant Gromov-Hausdorff convergence topology (or, equivalently, in the length function topology). It is known that $\overline{\mathrm{cv}}\left(F_{N}\right)$ consists of all very small minimal isometric actions of $F_{N}$ on $\mathbb{R}$-trees. The projectivization of $\overline{\mathrm{cv}}\left(F_{N}\right)$ provides a free group analogue of Thurston's compactification of Teichmüller space.

As an application, using the intersection graph determined by the intersection form, we show that several natural analogues of the curve complex in the free group context have infinite diameter.

20F65; 57M99, 37B99, 37D99

\section{Introduction}

The notion of intersection number plays a crucial role in the study of Teichmüller space, mapping class groups and their applications to 3-manifold topology. Thurston [37] extended the notion of a geometric intersection number between two free homotopy classes of closed curves on a surface to the notion of a geometric intersection number between two measured geodesic laminations. Indeed, this intersection number is a central concept in the study of Thurston's compactification of the Teichmüller space, as well as in the study of the dynamics and geometry of surface homeomorphisms. Bonahon [6] extended this notion of geometric intersection number to the case of two geodesic currents on the surface. Bonahon also constructed [7] a mapping class group equivariant embedding of Thurston's compactification of the Teichmüller space into the space of projectivized geodesic currents.

Culler and Vogtmann introduced in [13] a free-group analogue of Teichmüller space, which has been termed Outer space by Shalen and is denoted here by $\operatorname{CV}\left(F_{N}\right)$ (where 
$F_{N}$ is a free group of finite rank $N \geq 2$ ). Whereas points in Teichmüller space can be thought of as free and discrete isometric actions of the surface group on $\mathbb{H}^{2}$, points in $\mathrm{CV}\left(F_{N}\right)$ are represented by minimal free and discrete isometric actions of $F_{N}$ on $\mathbb{R}$-trees with normalized covolume (that is, where the quotient graph has volume 1). One also often works with the unprojectivized Outer space $\operatorname{cv}\left(F_{N}\right)$, which contains a copy of $\mathrm{CV}\left(F_{N}\right)$ and consists of all actions of the above type with arbitrary covolume. More details are given in Section 2 below.

Let $\overline{\operatorname{cv}}\left(F_{N}\right)$ be the closure of $\operatorname{cv}\left(F_{N}\right)$ in the equivariant Gromov-Hausdorff topology. It is known (see Cohen and Lustig [9] and Bestvina and Feighn [2]) that $\overline{\mathrm{cv}}\left(F_{N}\right)$ consists precisely of all the minimal very small nontrivial isometric actions on $F_{N}$ on $\mathbb{R}$-trees (see Section 2 for definitions). Projectivizing $\overline{\mathrm{cv}}\left(F_{N}\right)$ gives a Thurston-type compactification $\overline{\mathrm{CV}}\left(F_{N}\right)=\mathrm{CV}\left(F_{N}\right) \cup \partial \mathrm{CV}\left(F_{N}\right)$ of Outer space, where $\partial \mathrm{CV}\left(F_{N}\right)$ is the Thurston boundary of $\mathrm{CV}\left(F_{N}\right)$. The outer automorphism group $\operatorname{Out}\left(F_{N}\right)$ of $F_{N}$ acts on $\mathrm{CV}\left(F_{N}\right)$ and $\overline{\mathrm{CV}}\left(F_{N}\right)$ in very close analogy to the action of the mapping class group on Teichmüller space and its Thurston compactification. One can regard $\partial \operatorname{cv}\left(F_{N}\right)=\overline{\operatorname{cv}}\left(F_{N}\right)-\operatorname{cv}\left(F_{N}\right)$ as the boundary of $\operatorname{cv}\left(F_{N}\right)$. The Thurston boundary $\partial \mathrm{CV}\left(F_{N}\right)$ is obtained by projectivizing $\partial \operatorname{cv}\left(F_{N}\right)$.

The structure of Outer space and of $\operatorname{Out}\left(F_{N}\right)$ is more complicated than that of the Teichmüller space and the mapping class group. In large part this is due to the fact most free group automorphisms are not "geometric", in the sense that they are not induced by surface homeomorphisms. Although finite dimensional, $\mathrm{CV}\left(F_{N}\right)$ is not a manifold, and hence none of the tools from complex analysis which are so useful for surfaces can be directly carried over into the free group world. Moreover, while the topological and homotopy properties of Outer space are fairly well understood, very little is known about the geometry of $\mathrm{CV}\left(F_{N}\right)$. One of the reasons for this has been the lack, until recently, of a good geometric intersection theory in the Outer space context.

A geodesic current is a measure-theoretic generalization of the notion of a conjugacy class of a group element or of a free homotopy class of a closed curve on a surface (see Definition 5.4 below). Much of the motivation for studying currents comes from the work of Bonahon about geodesic currents on hyperbolic surfaces [6;7]. The space $\operatorname{Curr}\left(F_{N}\right)$ of all geodesic currents has a useful linear structure and admits a canonical $\operatorname{Out}\left(F_{N}\right)$-action. The space $\operatorname{Curr}\left(F_{N}\right)$ turns out to be a natural companion of the Outer space and contains additional valuable information about the geometry and dynamics of free group automorphisms. Examples of such applications can be found in Bonahon [8], Coulbois, Hilion and Lustig [12], Francaviglia [14], Kaimanovich, Kapovich and Schupp [22], Kapovich [23; 24; 25], Kapovich and Lustig [29], Kapovich and Nagnibeda [30], Martin [34] and other sources. 
In $[24 ; 32]$ we introduced a Bonahon-type, continuous, and $\operatorname{Out}\left(F_{N}\right)$-invariant geometric intersection form

$$
\langle,\rangle: \operatorname{cv}\left(F_{N}\right) \times \operatorname{Curr}\left(F_{N}\right) \rightarrow \mathbb{R}_{\geq 0} .
$$

The geometric intersection form $\langle$,$\rangle is \mathbb{R}_{>0}$-homogeneous with respect to the first argument, $\mathbb{R}_{\geq 0}$-linear with respect to the second argument and is $\operatorname{Out}\left(F_{N}\right)$-equivariant. This intersection form has the following crucial property in common with Bonahon's notion of an intersection number between two geodesic currents on a surface:

For any $\mathbb{R}$-tree $T \in \operatorname{cv}\left(F_{N}\right)$ and for any $g \in F_{N} \backslash\{1\}$ we have $\left\langle T, \eta_{g}\right\rangle=\|g\|_{T}$. Here $\eta_{g}$ is the counting current of $g$ (see Definition 5.6) and $\|g\|_{T}$ is the translation length of $g$ on the $\mathbb{R}$-tree $T$. Since the scalar multiples of all counting currents form a dense set in $\operatorname{Curr}\left(F_{N}\right)$, there is at most one continuous intersection form with the above properties, so that $\langle$,$\rangle is in fact canonical. Kapovich proved [24] that the intersection$ form $\langle$,$\rangle does not admit a "reasonable" continuous \operatorname{Out}\left(F_{N}\right)$-invariant symmetric extension to a map $\operatorname{Curr}\left(F_{N}\right) \times \operatorname{Curr}\left(F_{N}\right) \rightarrow \mathbb{R}$.

The main result of this paper is that the geometric intersection form $\langle$,$\rangle admits a$ continuous extension to the boundary of $\operatorname{cv}(F)$. We present a simplified form of this result here and refer to Theorem 6.1 below for a more detailed statement.

Theorem $\mathbf{A}$ Let $N \geq 2$. There exists a unique continuous map

$$
\langle,\rangle: \overline{\operatorname{cv}}\left(F_{N}\right) \times \operatorname{Curr}\left(F_{N}\right) \rightarrow \mathbb{R}_{\geq 0}
$$

which is $R_{\geq 0}$-homogeneous in the first argument, $R_{\geq 0}$-linear in the second argument, $\operatorname{Out}\left(F_{N}\right)$-invariant, and such that for every $T \in \overline{\mathrm{cv}}\left(F_{N}\right)$ and every $g \in F_{N} \backslash\{1\}$ we have

$$
\left\langle T, \eta_{g}\right\rangle=\|g\|_{T} .
$$

It is easy to see that the map $\langle$,$\rangle in Theorem A coincides with the intersection form$ from [24], when restricted to $\operatorname{cv}\left(F_{N}\right) \times \operatorname{Curr}\left(F_{N}\right)$.

Note that a very different and symmetric notion of an intersection number between two elements of $\overline{\mathrm{cv}}\left(F_{N}\right)$ was introduced and studied by Guirardel [18]. However, Guirardel's intersection number often takes on the value $\infty$, and it is fairly difficult to use.

A key ingredient in the proof of Theorem A is Proposition 4.1 below, which establishes a "Uniform Scaling Approximation Property" for points in $\overline{\mathrm{cv}}\left(F_{N}\right)$. It is clear that Proposition 4.1 should have further useful applications in the study of the boundary 
of the Outer space. The proof of Proposition 4.1 in turn relies on the Bounded BackTracking Property for very small actions of $F_{N}$ on $\mathbb{R}$-trees, established by Gaboriau, Jaeger, Levitt and Lustig [15].

Recall that for a closed hyperbolic surface $S$ the curve graph $\mathcal{C}(S)$ is defined as follows. The vertices of $\mathcal{C}(S)$ are free homotopy classes of essential simple closed curves on $S$. Two distinct vertices of $\mathcal{C}(S)$ are adjacent in $\mathcal{C}(S)$ if they can be realized by disjoint simple closed curves. The curve graph turned out to be a valuable tool in the study of the mapping class groups, of Kleinian groups and in various applications to 3-manifolds. Masur and Minsky [35] and Hempel [21] established that the curve graph has infinite diameter. Moreover, Masur and Minsky [35] proved that the curve graph is Gromov hyperbolic.

Algebraically, an essential simple closed curve $\alpha$ on $S$ determines a splitting of $G=\pi_{1}(S)$ as an amalgamated free product or an HNN-extension over the cyclic subgroup generated by $\alpha$ (the amalgamated free product case occurs if $\alpha$ is separating and the HNN-extension case occurs if $\alpha$ is nonseparating). Moreover, it is known (see Zieschang, Vogt and Coldewey [38]) that all splittings of $G$ over $\mathbb{Z}$ arise in this fashion.

In the free group context, it is often more natural to consider splittings over the trivial group rather than over $\mathbb{Z}$. Thus we define the free splitting graph $\mathcal{F}=\mathcal{F}\left(F_{N}\right)$ as follows. The vertices of $\mathcal{F}$ correspond to proper free product decompositions $F_{N}=A * B$, where $A \neq\{1\}, B \neq\{1\}$, where two such splittings are considered to be equal if their Bass-Serre trees are $F_{N}$-equivariantly isometric. Adjacency in $\mathcal{F}\left(F_{N}\right)$ corresponds to admitting a splitting of $F_{N}$ that is a common refinement of the two splittings in question. Informally, two distinct splittings $F_{N}=A * B$ and $F_{N}=A^{\prime} * B^{\prime}$ are adjacent in $\mathcal{F}\left(F_{N}\right)$ if there exists a free product decomposition $F_{N}=C_{1} * C_{2} * C_{3}$ such that either $A=C_{1} * C_{2}, B=C_{3}$ and $A^{\prime}=C_{1}, B^{\prime}=C_{2} * C_{3}$, or else $A^{\prime}=C_{1} * C_{2}, B^{\prime}=C_{3}$ and $A=C_{1}, B=C_{2} * C_{3}$. It is not hard to see that $\mathcal{F}_{N}$ is connected for $N \geq 3$. We also define the dual free splitting graph $\mathcal{F}^{*}=\mathcal{F}^{*}\left(F_{N}\right)$ as follows. The vertex set of $\mathcal{F}^{*}\left(F_{N}\right)$ is the same as the vertex set of $\mathcal{F}\left(F_{N}\right)$. Two vertices $T_{1}$ and $T_{2}$ of $\mathcal{F}^{*}\left(F_{N}\right)$ are adjacent in $\mathcal{F}^{*}\left(F_{N}\right)$ if there exists a nontrivial element $a \in F_{N}$ which is elliptic with respect to both $T$ and $T_{1}$, that is $\|a\|_{T_{1}}=\|a\|_{T_{2}}$ (this adjacency condition turns out be equivalent to requiring that there exist a primitive, ie a member of a free basis, element of $F_{N}$ that is elliptic for both $T_{1}$ and $T_{2}$ ). In the standard curve complex context, analogues of definitions of adjacency in $\mathcal{F}$ and $\mathcal{F}^{*}$ are essentially equivalent to the standard definition of adjacency in $\mathcal{C}(S)$. Namely, two nonisotopic simple closed curves define adjacent vertices of $\mathcal{C}(S)$ if and only if the corresponding cyclic splittings of $\pi_{1}(S)$ have a common refinement. Also, two such curves define vertices at distance $\leq 2$ in $\mathcal{C}(S)$ if and only if the corresponding 
splittings of $\pi_{1}(S)$ have a common nontrivial elliptic element. However, in the context of free groups $\mathcal{F}$ and $\mathcal{F}^{*}$ appear to be rather different objects, with distances in $\mathcal{F}^{*}$ often being much smaller than in $\mathcal{F}$.

We also introduce a key new object $\mathcal{I}\left(F_{N}\right)$ called the intersection graph of $F_{N}$. The graph $\mathcal{I}\left(F_{N}\right)$ is a bipartite graph with vertices of two kinds: projective classes $[T]$ of very small $F_{N}$-trees $T \in \overline{\mathrm{cv}}\left(F_{N}\right)$ and projective classes $[\mu]$ of nonzero currents $\mu \in \operatorname{Curr}\left(F_{N}\right)$. Two vertices $[T]$ and $[\mu]$ are adjacent in $\mathcal{I}\left(F_{N}\right)$ whenever $\langle T, \mu\rangle=0$. For $N \geq 3$ the graph $\mathcal{I}\left(F_{N}\right)$ has a large $\operatorname{Out}\left(F_{N}\right)$-invariant connected component $\mathcal{I}_{0}\left(F_{N}\right)$ that contains all projective classes of Bass-Serre trees $T$ corresponding to nontrivial free product decompositions of $F_{N}$ (this component also contains all the projective currents $\left[\eta_{a}\right]$ corresponding to primitive elements $a$ of $F_{N}$ ). Both $\mathcal{F}\left(F_{N}\right)$ and $\mathcal{F}^{*}\left(F_{N}\right)$ admit $\operatorname{Out}\left(F_{N}\right)$-equivariant Lipschitz maps into $\mathcal{I}_{0}\left(F_{N}\right)$ as do essentially all other reasonable analogues of the notion of a curve complex for free groups.

The results proved in Sections 7 and 8 of this paper can be summarized as follows:

Theorem B Let $N \geq 3$. Then the graphs $\mathcal{I}_{0}\left(F_{N}\right), \mathcal{F}\left(F_{N}\right)$ and $\mathcal{F}^{*}\left(F_{N}\right)$ have infinite diameter.

Moreover, if $Y_{N}$ is one of the above graphs and $\phi \in \operatorname{Out}\left(F_{N}\right)$ is an atoroidal iwip, ie $\phi$ is irreducible with irreducible powers (see Definition 7.3) and has no periodic conjugacy classes in $F_{N}$, then for any vertices $x, y$ of $Y_{N}$ we have

$$
\lim _{n \rightarrow \infty} d_{Y_{N}}\left(x, \phi^{n} y\right)=\infty .
$$

Recently Behrstock, Bestvina and Clay [1] obtained by different arguments an independent proof the conclusion of Theorem B for the complex $\mathcal{S}\left(F_{N}\right)$ which is quasi-isometric to $\mathcal{F}\left(F_{N}\right)$ (see Definition 8.4 and the subsequent discussion).

Our proof of Theorem B also shows that the "directions to infinity", given by Theorem B, corresponding to substantially different $\phi$, are distinct. Thus one can also show that if $\psi$ and $\phi$ are two elements of $\operatorname{Out}\left(F_{N}\right)$ such that they are irreducible with irreducible powers and without periodic conjugacy classes and such that the subgroup $\langle\phi, \psi\rangle$ is not virtually cyclic, then for any $x \in V Y_{N}$ and any sequences $n_{i} \rightarrow \infty, m_{i} \rightarrow \infty$ we have

$$
\lim _{i \rightarrow \infty} d_{Y_{N}}\left(\phi^{n_{i}} x, \psi^{m_{i}} x\right)=\infty .
$$

We also consider several natural variations of $\mathcal{F}\left(F_{N}\right)$ and $\mathcal{F}^{*}\left(F_{N}\right)$ and note that conclusions of Theorem B apply to them as well. Note that the intersection graph $\mathcal{I}\left(F_{N}\right)$ is not connected and it has other interesting connected components apart from 
$I_{0}\left(F_{N}\right)$. For example, for any $T \in \operatorname{cv}\left(F_{N}\right)$ and for any current $\mu$ with full support, $[T]$ and $[\mu]$ are isolated vertices of $\mathcal{I}\left(F_{N}\right)$. Similarly, if $\phi \in \operatorname{Out}\left(F_{N}\right)$ is an atoroidal iwip then for the "stable eigentree" $T(\phi)$ and "stable eigencurrent" $\mu(\phi)$ the pair $[T(\phi)],[\mu(\phi)]$ forms an isolated edge in $\mathcal{I}\left(F_{N}\right)$ (see Kapovich and Lustig [27] for details).

It seems plausible that the graphs $\mathcal{F}\left(F_{N}\right)$ and $\mathcal{F}^{*}\left(F_{N}\right)$ are not quasi-isometric. Investigating hyperbolicity properties of these graphs remains an interesting open problem for future study. It appears that $\mathcal{F}\left(F_{N}\right)$ is unlikely to be Gromov hyperbolic while $\mathcal{F}^{*}\left(F_{N}\right)$ does seem to have a chance for hyperbolicity. In particular, a reducible element of $\operatorname{Out}\left(F_{N}\right)$ always acts on $\mathcal{F}^{*}\left(F_{N}\right)$ with a bounded orbit while it seems possible for a reducible automorphism to act on $\mathcal{F}\left(F_{N}\right)$ with an unbounded orbit. Also, it seems that the orbits in $\mathcal{F}\left(F_{N}\right)$ of free abelian subgroups of $\operatorname{Out}\left(F_{N}\right)$ may produce quasi-flats, while similar orbits in $\mathcal{F}^{*}\left(F_{N}\right)$ have finite diameter.

The main result of this paper, Theorem A, seems to have the potential to become a important tool in the study of Outer space and $\operatorname{Out}\left(F_{N}\right)$ :

- We prove in Sections 7 and 8 of this paper that several free group analogues of the curve complex have infinite diameter.

- Ursula Hamenstädt had recently used [19] Theorem A as a crucial ingredient to prove that any nonelementary subgroup of $\operatorname{Out}\left(F_{N}\right)$, where $N \geq 3$, has infinite dimensional second bounded cohomology group (infinite dimensional space of quasi-morphisms). This in turn has an application to proving that any homomorphism from any lattice in a higher-rank semisimple Lie group to $\operatorname{Out}\left(F_{N}\right)$ has finite image.

- Very recently Bestvina and Feighn [4] used Theorem A as a key tool in the proof that for any finite collection $\phi_{1}, \ldots, \phi_{m} \in \operatorname{Out}\left(F_{N}\right)$ of iwip (irreducible with irreducible powers-see Definition 7.3) outer automorphisms of $F_{N}$ there exists a $\delta$-hyperbolic complex $X$ with an isometric $\operatorname{Out}\left(F_{N}\right)$-action where each $\phi_{i}$ acts with a positive translation length. Unlike the curve complex analogues discussed here in Sections 7 and 8, the Bestvina-Feighn construction is not intrinsically defined, but their result gives substantial hope and significant indication that some of these other more functorial and intrinsic analogues of the curve complex for free groups may be Gromov hyperbolic as well.

- The results of the new paper [4] of Bestvina and Feighn also imply that if $\phi \in \operatorname{Out}\left(F_{N}\right)$ is an iwip, then $\phi$ acts with positive asymptotic translation length on $\mathcal{F}\left(F_{N}\right)$ and on $\mathcal{F}^{*}\left(F_{N}\right)$. This means that when $Y_{N}$ is one of these two graphs, and $T \in V Y_{N}$ is an arbitrary vertex, then the orbit map $\mathbb{Z} \rightarrow Y_{N}$, $n \mapsto \phi^{n} T$, is a quasi-isometric embedding. 
- In a new preprint [26], we use Theorem A to construct domains of discontinuity for the action of subgroups of $\operatorname{Out}\left(F_{N}\right)$ on $\overline{\mathrm{CV}}\left(F_{N}\right)$ and on $\mathbb{P} \operatorname{Curr}\left(F_{N}\right)$.

- In another new preprint [28] we show that every subgroup of $\operatorname{Out}\left(F_{N}\right)$ which contains an atoroidal iwip and is not virtually cyclic, also contains a nonabelian free subgroup where every nontrivial element is an atoroidal iwip.

- Finally, in [27] we use Theorem A to characterize the situation where $\langle T, \mu\rangle=$ 0 . Specifically, we prove that for $T \in \overline{\operatorname{cv}}\left(F_{N}\right)$ and $\mu \in \operatorname{Curr}\left(F_{N}\right)$ we have $\langle T, \mu\rangle=0$ if and only if $\operatorname{supp}(\mu) \subseteq L^{2}(T)$. Here $\operatorname{supp}(\mu)$ is the support of $\mu$ and $L^{2}(T)$ is the dual algebraic lamination of $T$ (see Coulbois, Hilion and Lustig [10]). That result in turn is applied in [27] to the notions of a filling conjugacy class and a filling current as well as to obtain results about bounded translation equivalence in $F_{N}$. In [27] we also obtain a generalization of the length compactness result of Francaviglia [14]: we show that if $T \in \operatorname{cv}\left(F_{N}\right)$ and $\mu \in \operatorname{Curr}\left(F_{N}\right)$ is a current with full support (eg the Patterson-Sullivan current [30]) then for every $C>0$ the set $\left\{\phi \in \operatorname{Out}\left(F_{N}\right):\langle T, \phi \mu\rangle \leq C\right\}$ is finite and hence the set $\left\{\langle T, \phi \mu\rangle: \phi \in \operatorname{Out}\left(F_{N}\right)\right\} \subseteq \mathbb{R}$ is discrete.

Acknowledgments The authors are grateful to Gilbert Levitt, Thierry Coulbois, Mark Sapir and Saul Schleimer for useful and stimulating conversations that were very helpful in writing this paper. Our special thanks go to Saul Schleimer whose persistent questions inspired Sections 7 and 8 of this paper. The backwards iteration idea for the proof of Proposition 7.8 was suggested to us by Gilbert Levitt.

This paper grew out of a series of discussions that the authors had in October-November 2007 at MSRI Berkeley. We would like to thank MSRI and the organizers of the MSRI special semester in Geometric Group Theory for the financial support that made these discussions possible.

The first author was supported by the NSF grants DMS-0404991 and DMS-0603921. Both authors acknowledge the support of MSRI Berkeley a part of the special semester in Geometric Group Theory.

\section{Outer space and its closure}

We will only briefly recall the main definitions related to Outer space here. For a more detailed background information we refer the reader to Bestvina and Feighn [3], Cohen and Lustig [9], Culler and Vogtmann [13], Guirardel [17], Lustig [33] and other sources. 
Let $F_{N}$ be a free group of finite rank $N \geq 2$. Let $T$ be an $\mathbb{R}$-tree with an isometric action of $F_{N}$. For any $g \in F_{N}$, denote

$$
\|g\|_{T}=\inf _{x \in T} d_{T}(x, g x)=\min _{x \in T} d_{T}(x, g x) .
$$

The number $\|g\|_{T}$ is called the translation length of $g$.

Remark 2.1 Note that for all $m \in \mathbb{Z}$ we have

$$
\left\|g^{m}\right\|_{T}=|m| \cdot\|g\|_{T} .
$$

An isometric action of $F_{N}$ on an $\mathbb{R}$-tree $T$ is called minimal if $T$ has no proper $F_{N}$-invariant subtrees.

Definition 2.2 An isometric action of $F_{N}$ on an $\mathbb{R}$-tree $T$ action is called very small if:

(1) The stabilizer in $F_{N}$ of every nondegenerate arc in $T$ is cyclic (either trivial or infinite cyclic).

(2) The stabilizer in $F_{N}$ of every nondegenerate tripod is trivial.

(3) For every $g \in F_{N}, g \neq 1$ and every integer $n \neq 0$ if $g^{n}$ fixes some nondegenerate arc, then $g$ fixes that arc.

Thus free isometric actions of $F_{N}$ on $\mathbb{R}$-trees, and, more generally, actions with trivial arc stabilizers, are very small.

Definition 2.3 (Outer space and its closure) Let $N \geq 2$ be an integer.

(1) We denote by $\operatorname{cv}\left(F_{N}\right)$ the space of all minimal free and discrete isometric actions of $F_{N}$ on $\mathbb{R}$-trees. Two such actions of $F_{N}$ on trees $T$ and $T^{\prime}$ are identified in $\operatorname{cv}\left(F_{N}\right)$ if there exists an $F_{N}$-equivariant isometry between $T$ and $T^{\prime}$. The space $\operatorname{cv}\left(F_{N}\right)$ is called unprojectivized Outer space for $F_{N}$.

(2) Denote by $\overline{\mathrm{cv}}\left(F_{N}\right)$ the space of all minimal nontrivial very small isometric actions of $F_{N}$ on $\mathbb{R}$-trees. Again, two such actions are considered equal in $\overline{\operatorname{cv}}\left(F_{N}\right)$ if there exists an $F_{N}$-equivariant isometry between the two trees in question.

Note that if $T \in \operatorname{cv}\left(F_{N}\right)$ then the quotient $T / F_{N}$ is compact. It is known that every element $T \in \overline{\mathrm{cv}}\left(F_{N}\right)$ is uniquely identified by its translation length function $F_{N} \rightarrow \mathbb{R}, \quad g \mapsto\|g\|_{T}$. That is, for $T, T^{\prime} \in \overline{\mathrm{cv}}\left(F_{N}\right)$ we have $T=T^{\prime}$ if and only if $\|g\|_{T}=\|g\|_{T^{\prime}}$ for all $g \in F_{n}$. 
The spaces $\operatorname{cv}\left(F_{N}\right)$ and $\overline{\mathrm{cv}}\left(F_{N}\right)$ have several natural topologies that are all known to coincide [36]: the pointwise translation length function convergence topology, the equivariant Gromov-Hausdorff-Paulin convergence topology and the weak CW-topology (for the case of $\operatorname{cv}\left(F_{N}\right)$ ). In particular if $T_{n}, T \in \overline{\operatorname{cv}}\left(F_{N}\right)$ then $\lim _{n \rightarrow \infty} T_{n}=T$ if and only if for every $g \in F_{N}$ we have $\lim _{n \rightarrow \infty}\|g\|_{T_{n}}=\|g\|_{T}$. Note that $\operatorname{cv}\left(F_{N}\right) \subseteq \overline{\operatorname{cv}}\left(F_{N}\right)$. It is known that $\overline{\mathrm{cv}}\left(F_{N}\right)$ is precisely the closure of $\operatorname{cv}\left(F_{N}\right)$ (with respect to either of the above topologies).

There is a natural continuous action of $\operatorname{Out}\left(F_{N}\right)$ on $\overline{\mathrm{cv}}\left(F_{N}\right)$ that preserves $\operatorname{cv}\left(F_{N}\right)$, and which can be written from the left as well as from the right, using the convention $\phi T=T \phi^{-1}$ for $T \in \overline{\mathrm{cv}}\left(F_{N}\right)$ and $\phi \in \operatorname{Out}\left(F_{N}\right)$. At the translation-length-function level this action can be defined as follows. For $T \in \overline{\operatorname{cv}}\left(F_{N}\right)$ and $\hat{\varphi} \in \operatorname{Aut}\left(F_{N}\right)$ with image $\varphi \in \operatorname{Out}\left(F_{N}\right)$ we have

$$
\|g\|_{T \varphi}=\|g\|_{\varphi^{-1} T}=\|\hat{\varphi}(g)\|_{T}
$$

for any $g \in F_{N}$.

Definition 2.4 (Projectivized Outer space and its compactification) (1) For $N \geq 2$ one defines $\operatorname{CV}\left(F_{N}\right)=\operatorname{cv}\left(F_{N}\right) / \sim$, where $T_{1} \sim T_{2}$ whenever there exists an $F_{N^{-}}$ equivariant homothety between $T_{1}$ and $T_{2}$. Thus $T_{1} \sim T_{2}$ in $\operatorname{cv}\left(F_{N}\right)$ if and only if there is a constant $c>0$ such that $\|g\|_{T_{1}}=c \cdot\|g\|_{T_{2}}$ for all $g \in F_{N}$. The space $\mathrm{CV}\left(F_{N}\right)$, first introduced by Culler and Vogtmann [13], is called the projectivized Outer space or simply Outer space.

(2) Similarly, define $\overline{\mathrm{CV}}\left(F_{N}\right)=\overline{\mathrm{cv}}\left(F_{N}\right) / \sim$ where $\sim$ is again the above homothety relation. For $T \in \overline{\mathrm{cv}}\left(F_{N}\right)$ denote by $[T]$ the $\sim$-equivalence class of $T$.

(3) The spaces $\operatorname{CV}\left(F_{N}\right)$ and $\overline{\mathrm{CV}}\left(F_{N}\right)$ inherit the quotient topology from $\operatorname{cv}\left(F_{N}\right)$ and $\overline{\mathrm{cV}}\left(F_{N}\right)$. This makes the inclusion $\mathrm{CV}\left(F_{N}\right) \subseteq \overline{\mathrm{CV}}\left(F_{N}\right)$ into a topological embedding with dense image. Moreover, the space $\overline{\mathrm{CV}}\left(F_{N}\right)$ is compact and thus provides a natural compactification of $\operatorname{CV}\left(F_{N}\right)$. We also denote $\partial \mathrm{CV}\left(F_{N}\right)=\overline{\mathrm{CV}}\left(F_{N}\right) \backslash \mathrm{CV}\left(F_{N}\right)$ and call $\partial \mathrm{CV}\left(F_{N}\right)$ the Thurston boundary of $\mathrm{CV}\left(F_{N}\right)$.

The natural action of $\operatorname{Out}\left(F_{N}\right)$ on $\overline{\mathrm{cv}}\left(F_{N}\right)$ factors through to the action of $\operatorname{Out}\left(F_{N}\right)$ by homeomorphisms on $\overline{\mathrm{CV}}\left(F_{N}\right)$. Namely, for $\varphi \in \operatorname{Out}\left(F_{N}\right)$ and $T \in \overline{\mathrm{cV}}\left(F_{N}\right)$ we have $\varphi[T]=[\varphi T]$. This action of $\operatorname{Out}\left(F_{N}\right)$ on $\overline{\operatorname{CV}}\left(F_{N}\right)$ leaves $\operatorname{CV}\left(F_{N}\right)$ invariant, so that $\operatorname{Out}\left(F_{N}\right)$ acts on $\mathrm{CV}\left(F_{N}\right)$ as well.

Remark 2.5 There is a standard topological embedding $j: \mathrm{CV}\left(F_{N}\right) \rightarrow \operatorname{cv}\left(F_{N}\right)$ which is $\operatorname{Out}\left(F_{N}\right)$-equivariant that gives the identity on $\operatorname{CV}\left(F_{N}\right)$ when composed with the projection map $\operatorname{cv}\left(F_{N}\right) \rightarrow \operatorname{cv}\left(F_{N}\right) / \sim=\operatorname{CV}\left(F_{N}\right)$. Namely, $j([T])=T^{\prime}$, where 
$T^{\prime} \sim T$ and the quotient graph $T^{\prime} / F_{N}$ has volume 1 . One can alternatively think about elements of $\operatorname{cv}\left(F_{N}\right)$ as marked metric graph structures on $F_{N}$, as explained in more detail in Remark 5.2 below.

\section{Bounded back-tracking}

As before let $F_{N}$ be a free group of finite rank $N \geq 2$, and let $A$ be a free basis of $F_{N}$. We denote by $T_{A}$ the Cayley graph (which, of course, is a tree!) of $F_{N}$ with respect to $A$.

Let $T$ be an $\mathbb{R}$-tree with an isometric action of $F_{N}$, and consider a point $p \in T$. There is a unique $F_{N}$-equivariant map $i_{p}: T_{A} \rightarrow T$ which is linear on each edge of $T_{A}$, and which satisfies $i_{p}(1)=p$.

Definition 3.1 (Bounded back-tracking constant) The bounded back-tracking constant with respect to $A, T$ and $p$, denoted $\mathrm{BBT}_{p, A}(T)$, is the infimum of all constants $C>0$ such that for any $Q, R \in T_{A}$, the image $i_{p}([Q, R])$ of $[Q, R] \subseteq T_{A}$ is contained in the $C$-neighborhood of $\left[i_{p}(Q), i_{p}(R)\right]$.

A useful result of Gaboriau, Jaeger, Levitt and Lustig [15] states:

Proposition 3.2 Let $F_{N}$ be a finitely generated nonabelian free group with a minimal very small isometric action on an $\mathbb{R}$-tree $T$. Let $A$ be a free basis of $F_{N}$ and let $p \in T$.

Then we have

$$
\mathrm{BBT}_{p, A}(T) \leq \sum_{a \in A} d_{T}(p, a p) .
$$

In particular, $\mathrm{BBT}_{p, A}(T)<\infty$.

The following is an easy corollary of the definitions (see Lemma 3.1(b) of [15] or Lemma 3.1 of [11]):

Lemma 3.3 Let $F_{N}$ be a finitely generated nonabelian free group with a minimal very small isometric action on an $\mathbb{R}$-tree $T$. Let $A$ be a free basis of $F_{N}$ and let $p \in T$.

Suppose $\mathrm{BBT}_{p, A}(T)<C$. Then the following hold:

(1) Let $w \in F(A)$ be cyclically reduced. Then

$$
\left|\|w\|_{T}-d_{T}(p, w p)\right| \leq 2 C .
$$


(2) Let $u=u_{1} \ldots u_{m}$ be a freely reduced product of freely reduced words in $F=F(A)$, where $m \geq 1$. Then we have

$$
\left|d_{T}(p, u p)-\sum_{i=1}^{m} d_{T}\left(p, u_{i} p\right)\right| \leq 2 m C .
$$

(3) Suppose $u, u_{1}, \ldots, u_{m}$ are as in (2) and that, in addition, $u$ is cyclically reduced in $F(A)$. Then

$$
\left|\|u\|_{T}-\sum_{i=1}^{m} d_{T}\left(p, u_{i} p\right)\right| \leq 2 m C+2 C \leq 4 m C .
$$

(4) Suppose $u, u_{1}, \ldots, u_{m}$ are as in (2) and that, in addition, $u, u_{1}, \ldots, u_{m}$ are cyclically reduced in $F(A)$. Then

$$
\left|\|u\|_{T}-\sum_{i=1}^{m}\left\|u_{i}\right\|_{T}\right| \leq 6 m C .
$$

\section{Uniform approximation of $\mathbb{R}$-trees}

Let $A$ be a free basis of $F_{N}$. Recall that $T_{A}$ is the Cayley tree of $F_{N}$ with respect to $A$, where all edges of $T_{A}$ have length 1 . Thus $T_{A} \in \operatorname{cv}\left(F_{N}\right)$. For $g \in F_{N}$ we denote by $|g|_{A}$ the freely reduced length of $g$ with respect to $A$, and we denote by $\|g\|_{A}$ the cyclically reduced length of $g$ with respect to $A$. Thus $\|g\|_{A}=\|g\|_{T_{A}}$.

The following statement is a key ingredient in the proof of the continuity of our geometric intersection number. We believe that it will also turn out to be useful in other circumstances.

Proposition 4.1 (Uniform scaling approximation) Let $T \in \overline{\mathrm{cv}}\left(F_{N}\right)$, let $A$ be a free basis of $F_{N}$ and let $\epsilon>0$. Then there exists a neighborhood $U_{\epsilon}$ of $T$ in $\overline{\mathrm{cv}}\left(F_{N}\right)$, such that for every $w \in F_{N}$ and every $T_{1}, T_{2} \in U_{\epsilon}$ we have

$$
\left|\|w\|_{T_{1}}-\|w\|_{T_{2}}\right| \leq \epsilon\|w\|_{A} .
$$

Proof Choose $p \in T$. Let $C>0$ be such that $d_{T}(p, a p)<C / N$ for every $a \in A$, so that by Proposition 3.2 we have $\mathrm{BBT}_{p, A}(T)<C$. It suffices to prove the proposition for all sufficiently small $\epsilon$, and we will assume that $\epsilon>0$ satisfies $N \epsilon \leq C$.

Choose an integer $M>1$ so that $16 C / M<\epsilon / 2$. Let $0<\epsilon_{1}<\epsilon$ be such that $2 \epsilon_{1} / M \leq \epsilon / 2$. 
Using the equivariant Gromov-Hausdorff-Paulin topology on $\overline{\mathrm{cv}}\left(F_{N}\right)$ it follows that there exists a neighborhood $U_{\epsilon}$ of $T$ in $\overline{\mathrm{cv}}\left(F_{N}\right)$ such that for every $T^{\prime} \in U_{\epsilon}$ the following holds: There is some $p^{\prime} \in T^{\prime}$ such that for every $g \in F_{N}$ with $|g|_{A} \leq M$ we have

$$
\left|d_{T}(p, g p)-d_{T^{\prime}}\left(p^{\prime}, g p^{\prime}\right)\right| \leq \epsilon_{1} .
$$

Hence $\mathrm{BBT}_{p^{\prime}, A}\left(T^{\prime}\right) \leq \sum_{a \in A} d_{T^{\prime}}\left(p^{\prime}, a p^{\prime}\right)<C+N \epsilon_{1} \leq 2 C$. We will now verify that the neighborhood $U_{\epsilon}$ satisfies the requirements of the proposition.

Let $T_{1}, T_{2} \in U_{\epsilon}$ be arbitrary, and let $p_{1} \in T_{1}, p_{2} \in T_{2}$ be chosen as above. Let $w \in F(A)$ be a nontrivial cyclically reduced word such that $\|w\|_{A}$ is divisible by $M$. Put $m=\|w\|_{A} / M$. Thus $m \geq 1$ is an integer. Write $w$ as a freely reduced product $w=u_{1} \ldots u_{m}$ in $F(A)$, where $\left|u_{i}\right|_{A}=M$ for all $i=1, \ldots, m$.

Then, by the properties of the BBT-constant listed in Lemma 3.3 (specifically, by part (3)), we have for $j=1,2$,

$$
\left|\|w\|_{T_{j}}-\sum_{i=1}^{m} d_{T_{j}}\left(p_{j}, u_{i} p_{j}\right)\right| \leq 8 C m .
$$

Also, $(*)$ implies that for $j=1,2$ the inequality

$$
\left|\sum_{i=1}^{m} d_{T}\left(p, u_{i} p\right)-\sum_{i=1}^{m} d_{T_{j}}\left(p_{j}, u_{i} p_{j}\right)\right| \leq m \epsilon_{1}
$$

holds. This implies

$$
\left|\|w\|_{T_{1}}-\|w\|_{T_{2}}\right| \leq 16 C m+2 m \epsilon_{1}=\frac{16 C+2 \epsilon_{1}}{M}\|w\|_{A} \leq \epsilon\|w\|_{A} .
$$

Thus we have established that $(\dagger)$ holds for every $w \in F_{N}$ with $\|w\|_{A}$ divisible by $M$.

For the general case let $w \in F(A)$ be an arbitrary nontrivial cyclically reduced word. Since $\left\|w^{M}\right\|_{A}=M\|w\|_{A}$ is divisible by $M$, we already know that ( $\dagger$ ) holds for $w^{M}$. By dividing by $M$ both sides of the inequality ( $\dagger$ ) for $w^{M}$, we conclude that ( $\dagger$ ) holds for $w$ in view of Remark 2.1.

\section{Geodesic currents}

Let $\partial F_{N}$ be the hyperbolic boundary of $F_{N}$ (see Ghys and de la Harpe [16] for background information about word hyperbolic groups). We denote

$$
\partial^{2} F_{N}=\left\{\left(\xi_{1}, \xi_{2}\right): \xi_{1}, \xi_{2} \in \partial F_{N} \text { and } \xi_{1} \neq \xi_{2}\right\} .
$$


Also denote by $\sigma: \partial^{2} F_{N} \rightarrow \partial^{2} F_{N}$ the "flip" map defined as $\sigma:\left(\xi_{1}, \xi_{2}\right) \mapsto\left(\xi_{2}, \xi_{1}\right)$ for $\left(\xi_{1}, \xi_{2}\right) \in \partial^{2} F_{N}$.

Definition 5.1 (Simplicial charts) A simplicial chart on $F_{N}$ is an isomorphism $\alpha: F_{N} \rightarrow \pi_{1}(\Gamma, x)$, where $\Gamma$ is a finite connected graph without valence-one vertices, and where $x$ is a vertex of $\Gamma$.

A simplicial chart $\alpha$ on $F_{N}$ defines an $F_{N}$-equivariant quasi-isometry between $F_{N}$ (with any word metric) and the universal covering $\widetilde{\Gamma}$, equipped with the simplicial metric (ie every edge has length 1 ). Correspondingly, we get canonical $F_{N}$-equivariant homeomorphisms $\partial \alpha: \partial F_{N} \rightarrow \partial \widetilde{\Gamma}$ and $\partial^{2} \alpha: \partial^{2} F_{N} \rightarrow \partial^{2} \widetilde{\Gamma}$, that do not depend on the choice of a word metric for $F_{N}$. If $\alpha$ is fixed, we will usually use these homeomorphisms to identify $\partial F_{N}$ with $\partial \widetilde{\Gamma}$ and $\partial^{2} F_{N}$ with $\partial^{2} \widetilde{\Gamma}$ without additional comment.

Remark 5.2 (a) Combinatorially, we adopt Serre's convention regarding graphs. Thus every edge $e \in E \Gamma$ comes equipped with the inverse edge $e^{-1}$, such that $e \neq e^{-1}$ and $\left(e^{-1}\right)^{-1}=e$. Moreover, for every $e \in E \Gamma$, the initial vertex of $e$ is the terminal vertex of $e^{-1}$ and the terminal vertex of $e$ is the initial vertex of $e^{-1}$. An orientation on $\Gamma$ is a partition $E \Gamma=E^{+} \Gamma \sqcup E^{-} \Gamma$ such that for every $e \in E \Gamma$ one of the edges $e, e^{-1}$ belongs to $E^{+} \Gamma$ and the other belongs to $E^{-} \Gamma$.

(b) Any simplicial chart $\alpha: F_{N} \rightarrow \pi_{1}(\Gamma, x)$ defines a finite-dimensional open cell in $\operatorname{cv}\left(F_{N}\right)$ and a finite-dimensional open simplex in $\mathrm{CV}\left(F_{N}\right)$. More precisely, let $L$ be a metric graph structure on $\Gamma$, that is, a map $L: E \Gamma \rightarrow R_{>0}$ such that $L(e)=L\left(e^{-1}\right)$ for every edge $e \in E \Gamma$. Then we can pull back $L$ to $\widetilde{\Gamma}$ by giving every edge in $\widetilde{\Gamma}$ the same length as that of its projection in $\Gamma$. Let $d_{L}$ be the resulting metric on $\widetilde{\Gamma}$, which makes $\widetilde{\Gamma}$ into an $\mathbb{R}$-tree. The action of $F_{N}$ on this tree, defined via $\alpha$, is a deck transformation action and thus minimal, free and discrete. Hence this action defines a point in $\operatorname{cv}\left(F_{N}\right)$. Varying the metric structure $L$ on $\Gamma$ produces an open cone $\Delta(\alpha) \subseteq \operatorname{cv}\left(F_{N}\right)$ in $\operatorname{cv}\left(F_{N}\right)$, which is homeomorphic to the positive open cone in $\mathbb{R}^{m}$. Here $m$ is the number of topological edges of $\Gamma$, that is, $m=\frac{1}{2} \# E \Gamma$. Thus we can think of a simplicial chart $\alpha: F_{N} \rightarrow \pi_{1}(\Gamma, x)$ as defining a local "coordinate patch" on $\operatorname{cv}\left(F_{N}\right)$.

(c) If we require the sum of the lengths of all the topological edges of $\Gamma$ to be equal to 1 , we get a subset $\Delta^{\prime}(\alpha)$ of $\operatorname{cv}\left(F_{N}\right)$ that is homeomorphic to an open simplex of dimension $m-1$. This subset $\Delta^{\prime}(\alpha)$ belongs to the subset $j\left(\mathrm{CV}\left(F_{N}\right)\right)$ defined in Remark 2.5, and hence projects homeomorphically to its image in $\mathrm{CV}\left(F_{N}\right)$.

(d) Moreover, the union of open cones $\Delta(\alpha)$ over all simplicial charts $\alpha$ is equal to $\operatorname{cv}\left(F_{N}\right)$, and this union is a disjoint union. Additionally, every point of $\operatorname{cv}\left(F_{N}\right)$ 
belongs to only a finite number of closures $\bar{\Delta}(\alpha)$ of such open cones. Similarly, the copy $j\left(\mathrm{CV}\left(F_{N}\right)\right)$ of $\mathrm{CV}\left(F_{N}\right)$ in $\operatorname{cv}\left(F_{N}\right)$ is the disjoint union of the open simplices $\Delta^{\prime}(\alpha)$ over all simplicial charts $\alpha$, and the closures of these open simplices in $\operatorname{cv}\left(F_{N}\right)$ form a locally finite cover of $j\left(\mathrm{CV}\left(F_{N}\right)\right)$.

Definition 5.3 (Cylinders) Let $\alpha: F_{N} \rightarrow \pi_{1}(\Gamma, x)$ be a simplicial chart on $F_{N}$. For a nontrivial reduced edge-path $\gamma$ in $\widetilde{\Gamma}$ denote by $\operatorname{Cyl}_{\widetilde{\Gamma}}(\gamma)$ the set of all $\left(\xi_{1}, \xi_{2}\right) \in \partial^{2} F_{N}$ such that the bi-infinite geodesic from $\widetilde{\alpha}\left(\xi_{1}\right)$ to $\widetilde{\alpha}\left(\xi_{2}\right)$ contains $\gamma$ as a subpath.

We call $\operatorname{Cyl}_{\widetilde{\Gamma}}(\gamma) \subseteq \partial^{2} F_{N}$ the two-sided cylinder corresponding to $\gamma$.

It is easy to see that $\mathrm{Cyl}_{\widetilde{\Gamma}}(\gamma) \subseteq \partial^{2} F_{N}$ is both compact and open. Moreover, the collection of all such cylinders, where $\gamma$ varies over all nontrivial reduced edge-paths in $\tilde{\Gamma}$, forms a basis of open sets in $\partial^{2} F_{N}$.

Definition 5.4 (Geodesic currents) A geodesic current (or simply current) on $F_{N}$ is a positive Radon measure on $\partial^{2} F_{N}$ which is $F_{N}$-invariant and $\sigma$-invariant. The set of all geodesic currents on $F_{N}$ is denoted by $\operatorname{Curr}\left(F_{N}\right)$. The set $\operatorname{Curr}\left(F_{N}\right)$ is endowed with the weak-* topology. This makes $\operatorname{Curr}\left(F_{N}\right)$ into a locally compact space.

Specifically, let $\alpha: F_{N} \rightarrow \pi_{1}(\Gamma, x)$ be a simplicial chart on $F_{N}$. Let $\mu_{n}, \mu \in \operatorname{Curr}\left(F_{N}\right)$. It is not hard to show [24] that $\lim _{n \rightarrow \infty} \mu_{n}=\mu$ in $\operatorname{Curr}\left(F_{N}\right)$ if and only if for every nontrivial reduced edge-path $\gamma$ in $\widetilde{\Gamma}$ we have

$$
\lim _{n \rightarrow \infty} \mu_{n}\left(\mathrm{Cyl}_{\widetilde{\Gamma}}(\gamma)\right)=\mu\left(\mathrm{Cyl}_{\widetilde{\Gamma}}(\gamma)\right) .
$$

Let $\mu \in \operatorname{Curr}\left(F_{N}\right)$ and let $v$ be a nontrivial reduced edge-path in $\Gamma$. Denote

$$
\langle v, \mu\rangle_{\alpha}:=\mu\left(\mathrm{Cyl}_{\tilde{\Gamma}}(\gamma)\right),
$$

where $\gamma$ is any edge-path in $\tilde{\Gamma}$ that is labelled by $v$. Since $\mu$ is $F_{N}$-invariant, this definition does not depend on the choice of the lift $\gamma$ of $v$.

There is a natural continuous left-action of $\operatorname{Aut}\left(F_{N}\right)$ on $\operatorname{Curr}\left(F_{N}\right)$ by linear transformations. Namely, let $\psi \in \operatorname{Aut}\left(F_{N}\right)$. Then $\psi$ is a quasi-isometry of $F_{N}$ and hence $\psi$ induces a homeomorphism $\partial \psi$ of $\partial F_{N}$ and hence a homeomorphism $\partial^{2} \psi: \partial^{2} F_{N} \rightarrow$ $\partial^{2} F_{N}$. Then for $\mu \in \operatorname{Curr}\left(F_{N}\right)$ and $S \subseteq \partial^{2} F_{N}$ put

$$
(\psi \mu)(S):=\mu\left(\partial^{2} \psi^{-1} S\right) .
$$

It is not hard to check [24] that $\psi \mu$ is indeed a geodesic current. Moreover, the group of inner automorphisms $\operatorname{Inn}\left(F_{N}\right)$ is contained in the kernel of the action of $\operatorname{Aut}\left(F_{N}\right)$ on $\operatorname{Curr}\left(F_{N}\right)$. Therefore this action factors through to a continuous action of $\operatorname{Out}\left(F_{N}\right)$ on $\operatorname{Curr}\left(F_{N}\right)$. 
Notation 5.5 (1) For any $g \in F_{N} \backslash\{1\}$ denote by $g^{\infty}=\lim _{n \rightarrow \infty} g^{n}$ and $g^{-\infty}=$ $\lim _{n \rightarrow \infty} g^{n}$ the two distinct limit points in $\partial F_{N}$. Hence one obtains $\left(g^{-\infty}, g^{\infty}\right) \in$ $\partial^{2} F_{N}$.

(2) For any $g \in F_{N}$ we will denote by $[g]_{F_{N}}$ or just by $[g]$ the conjugacy class of $g$ in $F_{N}$.

Definition 5.6 (Counting and rational currents) (1) Let $g \in F_{N}$ be a nontrivial element that is not a proper power in $F_{N}$. Set

$$
\eta_{g}=\sum_{h \in[g]}\left(\delta_{\left(h^{-\infty}, h^{\infty}\right)}+\delta_{\left(h^{\infty}, h^{-\infty}\right)}\right)
$$

where $\delta_{\left(h^{-\infty}, h^{\infty}\right)}$ denotes as usually the atomic Dirac (or "counting") measure concentrated at the point $\left(h^{-\infty}, h^{\infty}\right)$.

Let $\mathcal{R}(g)$ be the collection of all $F_{N}$-translates of $\left(g^{-\infty}, g^{\infty}\right)$ and $\left(g^{\infty}, g^{-\infty}\right)$ in $\partial^{2} F_{N}$. This gives

$$
\eta_{g}=\sum_{(x, y) \in \mathcal{R}(g)} \delta_{(x, y)},
$$

and hence $\eta_{g}$ is $F_{N}$-invariant and flip-invariant, that is $\eta_{g} \in \operatorname{Curr}\left(F_{N}\right)$.

(2) Let $g \in F_{N} \backslash\{1\}$ be arbitrary. Write $g=f^{m}$ where $m \geq 1$ and $f \in F_{N}$ is not a proper power, and define

$$
\eta_{g}:=m \cdot \eta_{f}
$$

We call $\eta_{g} \in \operatorname{Curr}\left(F_{N}\right)$ the counting current given by $g$. Nonnegative scalar multiples of counting currents are called rational currents.

It is easy to see that if $[g]=[h]$ then $\eta_{g}=\eta_{h}$ and $\eta_{g}=\eta_{g}-1$. Thus $\eta_{g}$ depends only on the conjugacy class of $g$ and we will sometimes denote $\eta_{[g]}:=\eta_{g}$. Moreover, it is not hard to check [24] that for $\varphi \in \operatorname{Out}\left(F_{N}\right)$ and $g \in F_{N} \backslash\{1\}$ we have $\varphi \eta_{[g]}=\eta_{\varphi[g]}$. One can also give a more explicit combinatorial description of the counting current $\eta_{g}$ in terms of counting the numbers of occurrences of freely reduced words in a "cyclic word" $w$ representing $g$ (with respect to some fixed free basis of $F_{N}$ ). We refer the reader to [24] for details.

Proposition $5.7[23 ; 24]$ The set of all rational currents is dense in the space $\operatorname{Curr}\left(F_{N}\right)$.

Definition 5.8 (Projectivized space of geodesic currents) Let $N \geq 2$. We define

$$
\mathbb{P} \operatorname{Curr}\left(F_{N}\right)=\operatorname{Curr}\left(F_{N}\right) \backslash\{0\} / \sim
$$


where two currents $\mu_{1}, \mu_{2} \in \operatorname{Curr}\left(F_{N}\right) \backslash\{0\}$ satisfy $\mu_{1} \sim \mu_{2}$ if there is some constant $c>0$ such that $\mu_{2}=c \mu_{1}$. For a nonzero current $\mu \in \operatorname{Curr}\left(F_{N}\right)$ denote by $[\mu]$ the projective class of $\mu$, that is, the $\sim$-equivalence class of $\mu$.

The quotient set $\mathbb{P} \operatorname{Curr}\left(F_{N}\right)$ inherits the quotient topology from $\operatorname{Curr}\left(F_{N}\right)$ as well as a continuous action of $\operatorname{Out}\left(F_{N}\right)$. The space $\mathbb{P} \operatorname{Curr}\left(F_{N}\right)$ is called the projectivized space of geodesic currents (or simply space of projectivized currents) on $F_{N}$.

It is known $[23 ; 24]$ that $\mathbb{P} \operatorname{Curr}\left(F_{N}\right)$ is compact.

\section{The intersection form}

In this section we will prove the main result of this paper, whose slightly simplified version was stated in the Introduction as Theorem A. We state our result now in its full strength, using the terminology introduced in the previous sections.

\subsection{Statement of the main result}

Theorem 6.1 Let $N \geq 2$ be an integer. There exists a unique geometric intersection form

$$
\langle,\rangle: \overline{\mathrm{cv}}\left(F_{N}\right) \times \operatorname{Curr}\left(F_{N}\right) \rightarrow \mathbb{R}_{\geq 0}
$$

with the following properties.

(1) The function $\langle$,$\rangle is continuous.$

(2) The function $\langle$,$\rangle is R_{\geq 0}$-homogeneous in the first argument. Namely, for any $T \in \overline{\operatorname{cv}}\left(F_{N}\right), \mu \in \operatorname{Curr}\left(F_{N}\right)$ and $\lambda \geq 0$ we have

$$
\langle\lambda T, \mu\rangle=\lambda\langle T, \mu\rangle .
$$

(3) The function $\langle$,$\rangle is R_{\geq 0}$-linear in the second argument. Namely, for any $T \in \overline{\operatorname{cv}}\left(F_{N}\right), \mu_{1}, \mu_{2} \in \operatorname{Curr}\left(F_{N}\right) \lambda_{1}, \lambda_{2} \geq 0$ we have

$$
\left\langle T, \lambda_{1} \mu_{1}+\lambda_{2} \mu_{2}\right\rangle=\lambda_{1}\left\langle T, \mu_{1}\right\rangle+\lambda_{2}\left\langle T, \mu_{2}\right\rangle .
$$

(4) The function $\langle$,$\rangle is \operatorname{Out}\left(F_{N}\right)$-invariant: for any $T \in \overline{\mathrm{cv}}\left(F_{N}\right), \mu \in \operatorname{Curr}\left(F_{N}\right)$ and $\varphi \in \operatorname{Out}\left(F_{N}\right)$ we have

$$
\langle\varphi T, \varphi \mu\rangle=\langle T, \mu\rangle .
$$

(5) For any $T \in \overline{\mathrm{cv}}\left(F_{N}\right)$ and any $g \in F_{N}$, with associated counting current $\eta_{g} \in$ $\operatorname{Curr}\left(F_{N}\right)$, we have

$$
\left\langle T, \eta_{g}\right\rangle=\|g\|_{T}
$$


(6) Let $\alpha: F \rightarrow \pi_{1}(\Gamma, x)$ be a simplicial chart on $F$ and let $L: E \Gamma \rightarrow \mathbb{R}_{>0}$ be a metric graph structure on $\Gamma$ and let $T \in \operatorname{cv}(F)$ be the tree corresponding to the pullback of $L$ to $\widetilde{\Gamma}$, with the action of $F_{N}$ on $T$ via $\alpha$. Then for any $\mu \in \operatorname{Curr}\left(F_{N}\right)$ we have

$$
\langle\widetilde{\Gamma}, \mu\rangle=\sum_{e \in E^{+} \Gamma} L(e)\langle e, \mu\rangle_{\alpha},
$$

where $E \Gamma=E^{+} \Gamma \sqcup E^{-} \Gamma$ is an orientation on $\Gamma$.

Remark 6.2 (a) Note that conditions (1), (3) and (5) already imply that if such an intersection form exists, then it is unique. Indeed, recall that the set of rational currents is dense in $\operatorname{Curr}(F)$. Thus if $\mu \in \operatorname{Curr}(F)$ then there exists a sequence of rational currents $\lambda_{i} \eta_{g_{i}}$ such that $\mu=\lim _{i \rightarrow \infty} \lambda_{i} \eta_{g_{i}}$. Hence the continuity and linearity of the intersection form imply that

$$
\langle T, \mu\rangle=\lim _{i \rightarrow \infty} \lambda_{i}\left\|g_{i}\right\|_{T}
$$

Thus Theorem 6.1 implicitly implies that the above limit exists and does not depend on the choice of the sequence of rational currents converging to $\mu$.

(b) For the case of $\operatorname{cv}\left(F_{N}\right)$ the statement of Theorem 6.1 was already obtained in [24; 32], where we constructed the intersection form with the required properties on $\operatorname{cv}\left(F_{N}\right) \times \operatorname{Curr}\left(F_{N}\right)$. The main difficulty that had to be overcome in the present paper is to prove that intersection form admits a continuous "boundary" extension to a continuous map $\overline{\mathrm{cv}}\left(F_{N}\right) \times \operatorname{Curr}\left(F_{N}\right) \rightarrow \mathbb{R}$.

(c) Note that the $\operatorname{Out}\left(F_{N}\right)$-equivariance equality given in part (4) of Theorem 6.1 is equivalent to the formula

$$
\langle T \varphi, \mu\rangle=\langle T, \varphi \mu\rangle,
$$

as follows directly from the fact that the left side of this equation is equal to $\left\langle\varphi^{-1} T, \mu\right\rangle$ (see the definition of the $\operatorname{Out}\left(F_{N}\right)$-action in Section 2).

\subsection{The case of $\operatorname{cv}\left(F_{N}\right)$}

In $[24 ; 32]$ we established the statement of Theorem 6.1 for $\operatorname{cv}\left(F_{N}\right)$ :

Proposition-Definition 6.3 (Intersection form on $\operatorname{cv}\left(F_{N}\right)$ ) Let $N \geq 2$. There exists a unique map

$$
\langle,\rangle: \operatorname{cv}\left(F_{N}\right) \times \operatorname{Curr}\left(F_{N}\right) \rightarrow \mathbb{R}_{\geq 0}
$$

satisfying conditions (1)-(6) of Theorem 6.1 for arbitrary $T \in \operatorname{cv}(F)$.

For $T \in \operatorname{cv}\left(F_{N}\right)$ and $\mu \in \operatorname{Curr}\left(F_{N}\right)$ we call $\langle T, \mu\rangle$ the geometric intersection number of $T$ and $\mu$. 
Note, that, as we have seen in Remark 6.2, if $T \in \operatorname{cv}\left(F_{N}\right)$ and $\mu \in \operatorname{Curr}\left(F_{N}\right)$ is represented as $\mu=\lim _{i \rightarrow \infty} \lambda_{i} \eta_{g_{i}}$ for some $g_{i} \in F_{N}$ and $\lambda_{i} \geq 0$ then

$$
\langle T, \mu\rangle=\lim _{i \rightarrow \infty} \lambda_{i}\left\|g_{i}\right\|_{T}
$$

\subsection{Continuous extension of the intersection form to $\overline{\mathbf{c v}}\left(F_{N}\right)$}

The main tool to prove the existence of a continuous extension of the intersection form to $\overline{\mathrm{cv}}\left(F_{N}\right)$ will be Proposition 4.1. We first prove:

Proposition 6.4 Let $T \in \overline{\mathrm{cv}}\left(F_{N}\right)$ and $\mu \in \operatorname{Curr}\left(F_{N}\right)$ be such that $\mu=\lim _{i \rightarrow \infty} \lambda_{i} \eta_{g_{i}}$ for some $g_{i} \in F_{N}$ and $\lambda_{i} \geq 0$. Then the limit

$$
\lim _{i \rightarrow \infty} \lambda_{i}\left\|g_{i}\right\|_{T}
$$

exists and does not depend on the choice of the sequence $\lambda_{i} \eta_{g_{i}}$ of the rational currents that converges to $\mu$.

Proof Fix a free basis $A$ of $F_{N}$. Let $g_{i} \in F_{N}$ and $\lambda_{i} \geq 0$ be such that $\mu=$ $\lim _{i \rightarrow \infty} \lambda_{i} \eta_{g_{i}}$. We claim that $\lambda_{i}\left\|g_{i}\right\|_{T}$ is a Cauchy sequence of real numbers and hence has a finite limit.

Let $\epsilon>0$ be arbitrary. Choose $0<\epsilon_{1}<\epsilon$ such that $2 \epsilon_{1}\left(\left\langle T_{A}, \mu\right\rangle+\epsilon_{1}\right)+\epsilon_{1} \leq \epsilon$. Note that we allow for the possibility that $\mu=0$.

Let $U_{\epsilon_{1}}$ be the neighborhood of $T$ provided by Proposition 4.1. Choose a tree $T^{\prime} \in U_{\epsilon_{1}}$ such that $T^{\prime} \in \operatorname{cv}\left(F_{N}\right)$. Then $\left|\left\|g_{i}\right\|_{T}-\left\|g_{i}\right\|_{T^{\prime}}\right| \leq \epsilon_{1}\left\|g_{i}\right\|_{A}$ and hence

$$
\left|\lambda_{i}\left\|g_{i}\right\|_{T}-\lambda_{i}\left\|g_{i}\right\|_{T^{\prime}}\right| \leq \epsilon_{1} \lambda_{i}\left\|g_{i}\right\|_{A} .
$$

Recall that $\lim _{i \rightarrow \infty} \lambda_{i}\left\|g_{i}\right\|_{T^{\prime}}=\left\langle T^{\prime}, \mu\right\rangle$ and $\lim _{i \rightarrow \infty} \lambda_{i}\left\|g_{i}\right\|_{A}=\left\langle T_{A}, \mu\right\rangle$ since $T^{\prime}, T_{A}$ are in $\operatorname{cv}\left(F_{N}\right)$.

Thus there is $i_{0} \geq 1$ such that for every $i \geq i_{0}$ we have $\left|\lambda_{i}\left\|g_{i}\right\|_{T^{\prime}}-\left\langle T^{\prime}, \mu\right\rangle\right| \leq \epsilon_{1}$ and $\lambda_{i}\left\|g_{i}\right\|_{A} \leq\left\langle T_{A}, \mu\right\rangle+\epsilon_{1}$.

Thus for every $i \geq i_{0}$ we have

$$
\left|\lambda_{i}\left\|g_{i}\right\|_{T}-\left\langle T^{\prime}, \mu\right\rangle\right| \leq \epsilon_{1}\left(\left\langle T_{A}, \mu\right\rangle+\epsilon_{1}\right)+\epsilon_{1} .
$$

This implies that the sequence $\lambda_{i}\left\|g_{i}\right\|_{T}$ is bounded and, moreover, for any $i, j \geq i_{0}$

$$
\left|\lambda_{i}\left\|g_{i}\right\|_{T}-\lambda_{j}\left\|g_{j}\right\|_{T}\right| \leq 2\left(\epsilon_{1}\left(\left\langle T_{A}, \mu\right\rangle+\epsilon_{1}\right)+\epsilon_{1}\right) \leq \epsilon .
$$

Since $\epsilon>0$ was arbitrary, this shows that $\lambda_{i}\left\|g_{i}\right\|_{T}$ is a Cauchy sequence of real numbers and hence has a finite limit in $\mathbb{R}$. 
It is now clear that this limit does not depend on the choice of a sequence of rational currents $\lambda_{i} \eta_{g_{i}}$ such that $\lim _{i \rightarrow \infty} \lambda_{i} \eta_{g_{i}}=\mu$, since one can mix any two such sequences together to produce a new sequence of rational currents also limiting to $\mu$.

Proposition 6.4 implies that the following notion is well-defined:

Definition 6.5 (Intersection form on $\overline{\mathrm{cv}}\left(F_{N}\right)$ ) Let $T \in \overline{\mathrm{cv}}\left(F_{N}\right)$ and let $\mu \in \operatorname{Curr}\left(F_{N}\right)$. Put

$$
\langle T, \mu\rangle=\lim _{i \rightarrow \infty} \lambda_{i}\left\|g_{i}\right\|_{T}
$$

where $g_{i} \in F_{N}$ and $\lambda_{i} \geq 0$ are any such that $\mu=\lim _{i \rightarrow \infty} \lambda_{i} \eta_{g_{i}}$.

Note that the intersection number from Definition 6.5 agrees with the intersection number from Proposition-Definition 6.3 for arbitrary $T \in \operatorname{cv}\left(F_{N}\right)$ and $\mu \in \operatorname{Curr}\left(F_{N}\right)$.

Lemma 6.6 Let $A$ be a free basis of $F_{N}$. Let $T \in \overline{\mathrm{cv}}\left(F_{N}\right)$. Let $\epsilon>0$ and let $U_{\epsilon}$ be the neighborhood of $T$ in $\overline{\mathrm{cv}}\left(F_{N}\right)$ provided by Proposition 4.1. Then for any $T_{1}, T_{2} \in U_{\epsilon}$ and for any $v \in \operatorname{Curr}\left(F_{N}\right)$ have

$$
\left|\left\langle T_{1}, v\right\rangle-\left\langle T_{2}, v\right\rangle\right| \leq 2 \epsilon\left\langle T_{A}, v\right\rangle .
$$

Proof The statement is obvious if $v=0$ so we will assume that $v \neq 0$. Hence $\left\langle T_{A}, v\right\rangle>0$ and $\left\langle T_{0}, v\right\rangle>0$. Let $\epsilon_{1}>0$ be such that $\epsilon\left(\left\langle T_{A}, v\right\rangle+\epsilon_{1}\right)+2 \epsilon_{1} \leq 2 \epsilon\left\langle T_{A}, v\right\rangle$.

Let $v=\lim _{i \rightarrow \infty} \lambda_{i} \eta_{g_{i}}$ for some $g_{i} \in F_{N}$ and $\lambda_{i} \geq 0$. Choose $i_{0} \geq 1$ such that for every $i \geq i_{0}$

$$
\left|\left\langle T_{j}, v\right\rangle-\lambda_{i}\left\|g_{i}\right\|_{T_{j}}\right| \leq \epsilon_{1}, \quad \text { for } j=1,2
$$

and

$$
\left|\left\langle T_{A}, v\right\rangle-\lambda_{i}\left\|g_{i}\right\|_{A}\right| \leq \epsilon_{1} .
$$

Then for $i \geq i_{0}$ we have, by Proposition 4.1

$$
\begin{aligned}
\left|\left\langle T_{1}, v\right\rangle-\left\langle T_{2}, v\right\rangle\right| & \leq\left|\lambda_{i}\left\|g_{i}\right\|_{T_{1}}-\lambda_{i}\left\|g_{i}\right\|_{T_{2}}\right|+2 \epsilon_{1} \\
& \leq \epsilon \lambda_{i}\left\|g_{i}\right\|_{A}+2 \epsilon_{1} \leq \epsilon\left(\left\langle T_{A}, v\right\rangle+\epsilon_{1}\right)+2 \epsilon_{1} \leq 2 \epsilon\left\langle T_{A}, v\right\rangle .
\end{aligned}
$$

Proof of Theorem 6.1 We first show that the map $\langle\rangle:, \overline{\operatorname{cv}}\left(F_{N}\right) \times \operatorname{Curr}\left(F_{N}\right) \rightarrow \mathbb{R}_{\geq 0}$, given in Definition 6.5, is continuous.

Choose a free basis $A$ of $F_{N}$, and let $T \in \overline{\operatorname{cv}}\left(F_{N}\right), \mu \in \operatorname{Curr}\left(F_{N}\right)$ and $\epsilon>0$ be arbitrary.

Let $\epsilon_{1}>0$ be such that $4 \epsilon_{1}\left\langle T_{A}, \mu\right\rangle \leq \epsilon / 2$. Let $\epsilon_{2}>0$ be such that $2 \epsilon_{1} \epsilon_{2}+\epsilon_{2} \leq \epsilon / 2$. 
Let $U_{\epsilon_{1}} \subseteq \overline{\mathrm{cv}}\left(F_{N}\right)$ be the neighborhood of $T$ in $\overline{\mathrm{cv}}\left(F_{N}\right)$ provided by Proposition 4.1. Choose $T_{0} \in U_{\epsilon_{1}} \cap \mathrm{cv}\left(F_{N}\right)$.

Since $\langle\rangle:, \operatorname{cv}\left(F_{N}\right) \times \operatorname{Curr}\left(F_{N}\right) \rightarrow \mathbb{R}$ is continuous and since $T_{0}, T_{A} \in \operatorname{cv}\left(F_{N}\right)$, there exists a neighborhood $V$ of $\mu$ in $\operatorname{Curr}\left(F_{N}\right)$ such that for every $\mu^{\prime} \in V$ we have

and

$$
\begin{gathered}
\left|\left\langle T_{0}, \mu^{\prime}\right\rangle-\left\langle T_{0}, \mu\right\rangle\right| \leq \epsilon_{2} \\
\left|\left\langle T_{A}, \mu^{\prime}\right\rangle-\left\langle T_{A}, \mu\right\rangle\right| \leq \epsilon_{2} .
\end{gathered}
$$

Now let $T^{\prime} \in U_{\epsilon_{1}}$ and $\mu^{\prime} \in V$ be arbitrary. By Lemma 6.6 we have

$$
\begin{aligned}
\mid\left\langle T^{\prime}, \mu^{\prime}\right\rangle & -\langle T, \mu\rangle \mid \\
& \leq\left|\left\langle T^{\prime}, \mu^{\prime}\right\rangle-\left\langle T_{0}, \mu^{\prime}\right\rangle\right|+\left|\left\langle T_{0}, \mu^{\prime}\right\rangle-\left\langle T_{0}, \mu\right\rangle\right|+\left|\left\langle T_{0}, \mu\right\rangle-\langle T, \mu\rangle\right| \\
& \leq 2 \epsilon_{1}\left\langle T_{A}, \mu^{\prime}\right\rangle+\epsilon_{2}+2 \epsilon_{1}\left\langle T_{A}, \mu\right\rangle \\
& \leq 2 \epsilon_{1}\left\langle T_{A}, \mu\right\rangle+2 \epsilon_{1} \epsilon_{2}+\epsilon_{2}+2 \epsilon_{1}\left\langle T_{A}, \mu\right\rangle=4 \epsilon_{1}\left\langle T_{A}, \mu\right\rangle+2 \epsilon_{1} \epsilon_{2}+\epsilon_{2} \leq \epsilon .
\end{aligned}
$$

Since $\epsilon>0$ was arbitrary, this implies that $\langle\rangle:, \overline{\mathrm{cv}}\left(F_{N}\right) \times \operatorname{Curr}\left(F_{N}\right) \rightarrow \mathbb{R}$ is continuous at the point $(T, \mu)$. Since $(T, \mu) \in \overline{\operatorname{cv}}\left(F_{N}\right) \times \operatorname{Curr}\left(F_{N}\right)$ was arbitrary, it follows that $\langle\rangle:, \overline{\operatorname{cv}}\left(F_{N}\right) \times \operatorname{Curr}\left(F_{N}\right) \rightarrow \mathbb{R}$ is continuous, as required. This establishes part (1) of Theorem 6.1 .

The fact that parts (1)-(5) of Theorem 6.1 hold now follows by continuity from the same properties known to hold for $\langle\rangle:, \operatorname{cv}\left(F_{N}\right) \times \operatorname{Curr}\left(F_{N}\right) \rightarrow \mathbb{R}$. Part (6) of Theorem 6.1 only concerns $\mathbb{R}$-trees from $\operatorname{cv}\left(F_{N}\right)$ and is thus already known (see PropositionDefinition 6.3 above).

\section{The intersection form and iwip automorphisms of $\boldsymbol{F}_{N}$}

Notation 7.1 Note that if $T, T^{\prime} \in \overline{\operatorname{cv}}\left(F_{N}\right)$ and $\mu, \mu^{\prime} \in \operatorname{Curr}\left(F_{N}\right), \mu \neq 0, \mu^{\prime} \neq 0$ are such that $[T]=\left[T^{\prime}\right]$ and $[\mu]=\left[\mu^{\prime}\right]$ then $\langle T, \mu\rangle=0$ if and only if $\left\langle T^{\prime}, \mu^{\prime}\right\rangle=0$. Therefore for $x \in \overline{\mathrm{CV}}\left(F_{N}\right), y \in \mathbb{P} \operatorname{Curr}\left(F_{N}\right)$ we will write $\langle x, y\rangle=0$ if for some (or equivalently, for any) $T \in \overline{\operatorname{cv}}\left(F_{N}\right), \mu \in \operatorname{Curr}\left(F_{N}\right)$ with $[T]=x$ and $[\mu]=y$ we have $\langle T, \mu\rangle=0$.

Lemma 7.2 Let $\left[T_{n}\right],[T] \in \overline{\mathrm{CV}}\left(F_{N}\right)$ and let $\left[\mu_{n}\right] \in \mathbb{P} \operatorname{Curr}\left(F_{N}\right)$ be such that $\lim _{n \rightarrow \infty}\left[T_{n}\right]=[T]$ and $\lim _{n \rightarrow \infty}\left[\mu_{n}\right]=[\mu]$ and such that $\left\langle\left[T_{n}\right],\left[\mu_{n}\right]\right\rangle=0$ for every $n \geq 1$. Then

$$
\langle[T],[\mu]\rangle=0 .
$$


Proof There exist $r_{n} \geq 0$ and $c_{n} \geq 0$ such that $T=\lim _{n \rightarrow \infty} r_{n} T_{n}$ and $\mu=$ $\lim _{n \rightarrow \infty} c_{n} \mu_{n}$. By linearity of the intersection form, $\left\langle r_{n} T_{n}, c_{n} \mu_{n}\right\rangle=r_{n} c_{n}\left\langle T_{n}, \mu_{n}\right\rangle=0$. Hence by continuity (Theorem 6.1(1)) we have $\langle T, \mu\rangle=\lim _{n \rightarrow \infty}\left\langle r_{n} T_{n}, c_{n} \mu_{n}\right\rangle=0$, as required.

Definition 7.3 (IWIP) As in [31], we say that an outer automorphism $\varphi \in \operatorname{Out}\left(F_{N}\right)$ is irreducible with irreducible powers or an iwip if no conjugacy class of any nontrivial proper free factor of $F_{N}$ is mapped by a positive power of $\varphi$ to itself.

It is known that if such an iwip $\varphi$ is without periodic conjugacy classes, then $\varphi$ has a "North-South" dynamics for its induced actions on both, $\overline{\mathrm{CV}}\left(F_{N}\right)$ and $\mathbb{P} \operatorname{Curr}\left(F_{N}\right)$ :

Proposition 7.4 Let $N \geq 3$ and let $\varphi \in \operatorname{Out}\left(F_{N}\right)$ be irreducible with irreducible powers. Then the following hold:

(1) (Levitt-Lustig [31]) The action of $\varphi$ on $\overline{\mathrm{CV}}\left(F_{N}\right)$ has precisely two distinct fixed points, $\left[T_{+}\right]$and $\left[T_{-}\right]$, that both belong to $\partial \mathrm{CV}\left(F_{N}\right)$. Moreover, for any $[T] \neq\left[T_{-}\right]$in $\overline{\mathrm{CV}}\left(F_{N}\right)$ we have $\lim _{n \rightarrow \infty} \varphi^{n}[T]=\left[T_{+}\right]$. Similarly, for any $[T] \neq\left[T_{+}\right]$in $\overline{\mathrm{CV}}\left(F_{N}\right)$ we have $\lim _{n \rightarrow \infty} \varphi^{-n}[T]=\left[T_{-}\right]$.

(2) (Martin [34]) Suppose in addition that $\varphi$ has no periodic conjugacy classes in $F_{N}$. Then the action of $\varphi$ on $\mathbb{P} \operatorname{Curr}\left(F_{N}\right)$ has precisely two distinct fixed points $\left[\mu_{+}\right]$and $\left[\mu_{-}\right]$. Moreover, for any $[\mu] \neq\left[\mu_{-}\right]$in $\mathbb{P} \operatorname{Curr}\left(F_{N}\right)$ we have $\lim _{n \rightarrow \infty} \varphi^{n}[\mu]=\left[\mu_{+}\right]$. Similarly, for any $[\mu] \neq\left[\mu_{+}\right]$in $\mathbb{P} \operatorname{Curr}\left(F_{N}\right)$ we have $\lim _{n \rightarrow \infty} \varphi^{-n}[\mu]=\left[\mu_{-}\right]$.

Convention 7.5 For the remainder of this section, unless specified otherwise, let $N \geq 3$ and let $\varphi \in \operatorname{Out}\left(F_{N}\right)$ be irreducible with irreducible powers, and without periodic conjugacy classes. Let $\left[T_{+}\right],\left[T_{-}\right] \in \partial \mathrm{CV}\left(F_{N}\right)$ be the attracting and repelling fixed points for the (left) action of $\varphi$ on $\overline{\mathrm{CV}}\left(F_{N}\right)$. Similarly, let $\left[\mu_{+}\right],\left[\mu_{-}\right] \in \mathbb{P} \operatorname{Curr}\left(F_{N}\right)$ be the attracting and repelling fixed points for the action of $\varphi$ on $\mathbb{P} \operatorname{Curr}\left(F_{N}\right)$.

Remark 7.6 (1) We would like to alert the reader that the forward limit tree of $\varphi$, denoted in [12] and [33] by $T_{\varphi}$, is the tree $T_{-}$(and not $T_{+}$). This is due to the fact that in this paper $\varphi$ acts on $\mathbb{R}$-trees in $\overline{\mathrm{CV}}\left(F_{n}\right)$ from the left, while [12] and [33] in one considers the right-action (compare the discussion in Section 2).

(2) Some useful information about iwips and their induced action on Outer space has been worked out in detail in [33, Sections 4 and 5]. A summary of the most important facts is given in [12, Remark 5.5].

(3) An alternative proof (relying on the main result of [27]) for Proposition 7.7 below is given by Proposition 5.6 of [12]. 
Proposition 7.7 Let $\varphi, T_{ \pm}$and $\mu_{ \pm}$be as in Convention 7.5. Then

$$
\left\langle T_{-}, \mu_{+}\right\rangle \neq 0 \text { and }\left\langle T_{+}, \mu_{-}\right\rangle \neq 0 .
$$

Proof Let $\alpha: F_{N} \rightarrow \pi_{1}(\Gamma)$ be a marked graph structure on $F_{N}$, given by a train track map on $\Gamma$ that represents $\varphi$, with a metric structure $L$ on the edges of $\Gamma$ given by the Perron-Frobenius eigenvector of the transition matrix (see Bestvina and Handel [5] or Section 3 of Lustig [33] for a detailed exposition). Let $T=\widetilde{\Gamma} \in \operatorname{cv}\left(F_{N}\right)$ be the discrete $\mathbb{R}$-tree given by the universal covering of $\Gamma$, provided with the metric $d_{L}$ given by the lift of $L$, and with the action of $F_{N}$ coming from the marking $\alpha$.

Let $\lambda>1$ be the train track stretching constant for $\Gamma$ (ie the Perron-Frobenius eigenvalue of the transition matrix of the train track map). It is known (see, for example, Remark 5.4 of Lustig [33]) that $\lim _{n \rightarrow \infty}\left(1 / \lambda^{n}\right) \varphi^{-n} T=T_{-}$.

Let $g \in F_{N}, g \neq 1$ be arbitrary. Then there exists constants $C>1$ and $n_{0} \in \mathbb{N}$ such that for every $n \geq n_{0}$ we have

$$
\frac{1}{C} \lambda^{n} \leq\left\|\varphi^{n}(g)\right\|_{T} \leq C \lambda^{n} .
$$

The upper bound is derived in Section 3 of [33] before Remark 3.4: The inequality becomes an equality if $g$ is represented by a legal loop. The lower bound follows from the fact that every path in $\Gamma$ has an iterate (under the train track map) that is a legal composition of legal paths and INP's; see Bestvina and Handel [5].

Note that $\left\|\varphi^{n}(g)\right\|_{T}=\|g\|_{\varphi^{-n} T}$. It was proved by Martin [34] that $\lim _{n \rightarrow \infty}\left[\varphi^{n} \eta_{g}\right]=$ $\left[\mu_{+}\right]$and, moreover, that, after possibly multiplying $\mu_{+}$by a positive scalar, we have $\lim _{n \rightarrow \infty}\left(1 / \lambda^{n}\right) \eta_{\varphi^{n}(g)}=\mu_{+}$. We compute:

$$
\begin{aligned}
\left\langle T_{-}, \mu_{+}\right\rangle & =\lim _{n \rightarrow \infty}\left\langle\frac{1}{\lambda^{n}} \varphi^{-n} T, \frac{1}{\lambda^{n}} \eta_{\varphi^{n}}(g)\right\rangle \\
& =\lim _{n \rightarrow \infty} \frac{1}{\lambda^{2 n}}\left\langle\varphi^{-n} T, \eta_{\varphi^{n}(g)}\right\rangle \\
& =\lim _{n \rightarrow \infty} \frac{1}{\lambda^{2 n}}\left\langle T, \varphi^{n} \eta_{\varphi^{n}(g)}\right\rangle \\
& =\lim _{n \rightarrow \infty} \frac{1}{\lambda^{2 n}}\left\langle T, \eta_{\varphi^{2 n}(g)}\right\rangle \\
& =\lim _{n \rightarrow \infty} \frac{1}{\lambda^{2 n}}\left\|\varphi^{2 n}(g)\right\|_{T} \\
& \geq \lim _{n \rightarrow \infty} \frac{1}{\lambda^{2 n}} C \lambda^{2 n}=C>0
\end{aligned}
$$

Replacing $\varphi$ by $\varphi^{-1}$ we conclude that $\left\langle T_{+}, \mu_{-}\right\rangle>0$ as well. 
Proposition 7.8 Let $\left[T_{n}\right] \in \overline{\mathrm{CV}}\left(F_{N}\right)$ and $\left[\mu_{n}\right] \in \mathbb{P} \operatorname{Curr}\left(F_{N}\right)$ be sequences such that

$$
\left\langle\left[T_{n}\right],\left[\mu_{n}\right]\right\rangle=0
$$

for every $n \geq 1$. Then we have

$$
\lim _{n \rightarrow \infty}\left[T_{n}\right]=\left[T_{+}\right] \Longleftrightarrow \lim _{n \rightarrow \infty}\left[\mu_{n}\right]=\left[\mu_{+}\right] .
$$

Proof Let $\lim _{n \rightarrow \infty}\left[T_{n}\right]=\left[T_{+}\right]$. Suppose that $\lim _{n \rightarrow \infty}\left[\mu_{n}\right] \neq\left[\mu_{+}\right]$.

Since $\mathbb{P} \operatorname{Curr}\left(F_{N}\right)$ is compact, after passing to a subsequence we may assume that $\lim _{n \rightarrow \infty}\left[\mu_{n}\right]=[\mu]$ for some $[\mu] \neq\left[\mu_{+}\right]$in $\mathbb{P} \operatorname{Curr}\left(F_{N}\right)$. Note that by Lemma 7.2 we have $\left\langle\left[T_{+}\right],[\mu]\right\rangle=0$. Since $[\mu] \neq\left[\mu_{+}\right]$, part (2) of Proposition 7.4 implies that $\lim _{n \rightarrow \infty} \varphi^{-n}[\mu]=\left[\mu_{-}\right]$. Note that $\left[T_{+}\right]$is fixed by $\varphi^{-1}$, and that we have

$$
\left\langle\left[T_{+}\right], \varphi^{-n}[\mu]\right\rangle=\left\langle\varphi^{-n}\left[T_{+}\right], \varphi^{-n}[\mu]\right\rangle=\left\langle\left[T_{+}\right],[\mu]\right\rangle=0
$$

for every $n \geq 1$. Hence Lemma 7.2 implies $\left\langle\left[T_{+}\right],\left[\mu_{-}\right]\right\rangle=0$. This is to say that $\left\langle T_{+}, \mu_{-}\right\rangle=0$, yielding a contradiction with Proposition 7.7. Thus $\lim _{n \rightarrow \infty}\left[\mu_{n}\right]=\left[\mu_{+}\right]$. The argument for the other direction is completely symmetric.

\section{Curve complex analogues for free groups}

\subsection{The bipartite intersection graph}

Definition 8.1 (Intersection graph) Let $\mathcal{I}=\mathcal{I}\left(F_{N}\right)$ be a bipartite graph defined as follows. The vertex set of $\mathcal{I}$ is $V \mathcal{I}=\overline{\mathrm{CV}}\left(F_{N}\right) \cup \mathbb{P} \operatorname{Curr}\left(F_{N}\right)$. Two vertices $[T] \in \overline{\mathrm{CV}}\left(F_{N}\right)$ and $[\mu] \in \mathbb{P} \operatorname{Curr}\left(F_{N}\right)$ are connected by an edge in $\mathcal{I}$ if and only if $\langle[T],[\mu]\rangle=0$.

Since the intersection form is $\operatorname{Out}\left(F_{N}\right)$-invariant, the graph $\mathcal{I}\left(F_{N}\right)$ comes equipped with a natural action of $\operatorname{Out}\left(F_{N}\right)$ by graph automorphisms.

It is not hard to show that for $N \geq 3$ there is a single connected component $\mathcal{I}_{0}\left(F_{N}\right)$ of $\mathcal{I}\left(F_{N}\right)$ containing all $[T]$ for the Bass-Serre trees $T$ corresponding to all nontrivial free product decompositions of $F_{N}$. Moreover, the same connected component $\mathcal{I}_{0}\left(F_{N}\right)$ also contains $\eta_{a}$ for all nontrivial $a \in F_{N}$ that belong to some proper free factors of $F_{N}$. It is also not hard to show that $\mathcal{I}_{0}\left(F_{N}\right)$ is $\operatorname{Out}\left(F_{N}\right)$-invariant.

Note also that there are many connected components in this graph. Indeed, every vertex $[T] \in \mathrm{CV}\left(F_{N}\right)$ is an isolated point, and it follows from [12] that many pairs $([T],[\mu])$ form a single edge connected component, in particular all pairs $\left(\left[T_{+}\right],\left[\mu_{+}\right]\right)$as in Convention 7.5. 
Proposition 8.2 Let $\left[T_{n}\right],[T] \in \overline{\mathrm{CV}}\left(F_{N}\right)$ satisfy $[T] \neq\left[T_{+}\right]$and $\lim _{n \rightarrow \infty}\left[T_{n}\right]=\left[T_{+}\right]$, for $\left[T_{+}\right]$as in Convention 7.5. Then in the graph $\mathcal{I}$ we have

$$
\lim _{n \rightarrow \infty} d_{\mathcal{I}}\left(\left[T_{n}\right],[T]\right)=\infty
$$

Proof Suppose that the statement of the proposition fails. Then there exists a sequence $\left[T_{n}\right] \in \overline{\mathrm{CV}}\left(F_{N}\right)$ with $\lim _{n \rightarrow \infty}\left[T_{n}\right]=\left[T_{+}\right]$such that $\max _{n \geq 1} d_{\mathcal{I}}\left(\left[T_{n}\right],[T]\right)<\infty$. Among sequences $\left[T_{n}\right] \in \overline{\mathrm{CV}}\left(F_{N}\right)$ satisfying $\lim _{n \rightarrow \infty}\left[T_{n}\right]=\left[T_{+}\right]$and $\max _{n \geq 1} d_{\mathcal{I}}\left(\left[T_{n}\right],[T]\right)<$ $\infty$, choose a sequence $\left[T_{n}\right]$ such that $\max _{n \geq 1} d_{\mathcal{I}}\left(\left[T_{n}\right],[T]\right)$ is the smallest possible.

Let $D=\max _{n \geq 1} d_{\mathcal{I}}\left(\left[T_{n}\right],[T]\right)$. Suppose that $D>0$. Then, after passing to a further subsequence, we may assume that $\left[T_{n}\right] \neq[T]$ for every $n \geq 1$. Note that by definition of the graph $\mathcal{I}$, the numbers $D$ and $d_{\mathcal{I}}\left(\left[T_{n}\right],[T]\right)$ are positive even integers. By definition of $\mathcal{I}$ it follows that there exist $\left[T_{n}^{\prime}\right] \in \overline{\mathrm{CV}}\left(F_{N}\right)$ such that $d_{\mathcal{I}}\left(\left[T_{n}\right],\left[T_{n}^{\prime}\right]\right)=2$ and $d_{\mathcal{I}}\left(\left[T_{n}^{\prime}\right],[T]\right)=d_{\mathcal{I}}\left(\left[T_{n}\right],[T]\right)-2$. Hence, again by definition of $\mathcal{I}$, there exists a sequence $\left[\mu_{n}\right] \in \mathbb{P} \operatorname{Curr}\left(F_{N}\right)$ such that $\left\langle\left[T_{n}\right],\left[\mu_{n}\right]\right\rangle=0=$ $\left\langle\left[T_{n}^{\prime}\right],\left[\mu_{n}\right]\right\rangle$. Since $\lim _{n \rightarrow \infty}\left[T_{n}\right]=\left[T_{+}\right]$and $\left\langle\left[T_{n}\right],\left[\mu_{n}\right]\right\rangle=0$, Proposition $7.8 \mathrm{im}-$ plies that $\lim _{n \rightarrow \infty}\left[\mu_{n}\right]=\left[\mu_{+}\right]$. Since $\left\langle\left[T_{n}^{\prime}\right],\left[\mu_{n}\right]\right\rangle=0$, Proposition 7.8 then implies that $\lim _{n \rightarrow \infty}\left[T_{n}^{\prime}\right]=\left[T_{+}\right]$. Thus $\lim _{n \rightarrow \infty}\left[T_{n}^{\prime}\right]=\left[T_{+}\right]$and $\max _{n \geq 1} d_{\mathcal{I}}\left(\left[T_{n}^{\prime}\right],[T]\right)=$ $D-2<D=\max _{n \geq 1} d_{\mathcal{I}}\left(\left[T_{n}^{\prime}\right],[T]\right)$. This contradicts the minimality in the choice of $\left[T_{n}\right]$. Therefore we conclude $D=0$. Thus $0=\max _{n \geq 1} d_{\mathcal{I}}\left(\left[T_{n}\right],[T]\right)$ and hence $\left[T_{n}\right]=[T]$ for every $n \geq 1$. This contradicts the assumptions that $[T] \neq\left[T_{+}\right]$and that $\lim _{n \rightarrow \infty}\left[T_{n}\right]=\left[T_{+}\right]$.

Proposition 8.2 and Proposition 7.4 immediately imply:

Corollary 8.3 Let $\varphi,\left[T_{+}\right]$and $\left[T_{-}\right]$be as in Convention 7.5, and let $[T] \in \overline{\mathrm{CV}}\left(F_{N}\right)$ be such that $[T] \neq\left[T_{+}\right],\left[T_{-}\right]$. Then in the intersection graph $\mathcal{I}=\mathcal{I}\left(F_{N}\right)$ we have

$$
\lim _{n \rightarrow \infty} d_{\mathcal{I}}\left(\varphi^{n}[T],[T]\right)=\infty
$$

\subsection{Other curve complex analogues}

One can define several other natural free group analogues of the curve complex. Each of them will admit an $\operatorname{Out}\left(F_{N}\right)$-equivariant Lipschitz map into the intersection graph

\section{Definition 8.4 Let $N \geq 3$.}

(1) The free splitting graph $\mathcal{F}=\mathcal{F}\left(F_{N}\right)$ is a simple graph whose vertices are nontrivial splitting of $F_{N}$ as the fundamental group of a graph of groups with a single nonloop edge and trivial edge group (so algebraically they correspond to nontrivial free product 
decompositions of $F_{N}$ ). Two such splittings are considered equal if their Bass-Serre trees are $F_{N}$-equivariantly isometric, that is, if they equal as points $\operatorname{of} \operatorname{cv}\left(F_{N}\right)$. Two distinct splittings $T_{1}, T_{2} \in V \mathcal{F}\left(F_{N}\right)$ are adjacent in $\mathcal{F}$ if there exists a splitting of $F_{N}$ as the fundamental group of a graph of groups with two (nonloop) edges and trivial edge groups, such that $T_{1}$ is obtained by collapsing one edge of this graph of groups, and $T_{2}$ is obtained by collapsing the other edge.

(2) The cut graph $\mathcal{S}=\mathcal{S}\left(F_{N}\right)$ is a simple graph whose vertices are nontrivial splitting of $F_{N}$ as the fundamental group of a graph of groups with a single edge (possible a loop edge) and trivial edge group. Again, two such splittings are considered equal if their Bass-Serre trees are $F_{N}$-equivariantly isometric, that is, if they equal as points of $\operatorname{cv}\left(F_{N}\right)$. Two distinct splittings $T_{1}, T_{2} \in V \mathcal{F}\left(F_{N}\right)$ are adjacent in $\mathcal{F}$ if there exists a splitting of $F_{N}$ as the fundamental group of a graph of groups with two (nonloop) edges and trivial edge groups, such that $T_{1}$ is obtained by collapsing one edge of this graph of groups, and $T_{2}$ is obtained by collapsing the other edge.

(3) The dual free splitting graph $\mathcal{F}^{*}=\mathcal{F}^{*}\left(F_{N}\right)$ has the same vertex set as $\mathcal{F}\left(F_{N}\right)$. Two distinct vertices of $\mathcal{F}^{*}\left(F_{N}\right)$ corresponding to splittings $T_{1}$ and $T_{2}$ are adjacent whenever there exists a nontrivial element $g \in F_{N}$ such that $\|g\|_{T_{1}}=\|g\|_{T_{2}}=0$. One can show that this adjacency condition is equivalent to saying that there exists a nontrivial primitive (ie a member of a free basis of $F_{N}$ ) element $a$ of $F_{N}$ such that $\|a\|_{T_{1}}=\|a\|_{T_{2}}=0$.

(4) The dual cut graph $\mathcal{S}^{*}=\mathcal{S}^{*}\left(F_{N}\right)$ has the same vertex set as $\mathcal{S}\left(F_{N}\right)$. Two distinct vertices are adjacent in $\mathcal{S}^{*}\left(F_{N}\right)$ whenever the corresponding splittings of $F_{N}$ have some common nontrivial elliptic element.

(5) The ellipticity graph $\mathcal{Z}=\mathcal{Z}\left(F_{N}\right)$ is a bipartite graph. Its vertex set is the disjoint union of the vertex set of $\mathcal{S}\left(F_{N}\right)$ and the set of all $F_{N}$-conjugacy classes $[a]$ of nontrivial elements $a \in F_{N}$. A vertex $[a]$ is adjacent to a vertex $T$ whenever $a$ is an elliptic element with respect to $T$, that is $\|a\|_{T}=0$ (algebraically, this means that $a$ is conjugate to a vertex group element for the free product splitting $T$ ).

(6) The free factor graph $\mathcal{J}=\mathcal{J}\left(F_{N}\right)$ is a simple graph defined as follows: The vertex set of $\mathcal{J}$ is the set of conjugacy classes in $F_{N}$ of all free factors $A$ of $F_{N}$ such that $A \neq 1, A \neq F_{N}$. Two distinct vertices $x, y \in V \mathcal{J}$ are adjacent in $\mathcal{J}$ if and only if for some $A, B$ with $[A]=x$ and $[y]=B$ there exists $C \leq F_{N}$ such that $F_{N}=A * B * C$. Note that we allow the case where $C=1$.

(7) The dominance graph $\mathcal{D}=\mathcal{D}\left(F_{N}\right)$ is defined as follows. Put $V \mathcal{D}=V \mathcal{J}$. For distinct $x, y \in V \mathcal{D}$ we say that $x, y$ are adjacent in $\mathcal{D}$ if and only if there exist $A, B \leq F_{N}$ such that $x=[A], y=[B]$ and such that either $A \leq B$ or $B \leq A$. The 
dominance graph is precisely the one-skeleton of the "complex of free factors" $C F_{N}$ whose homotopy properties have been studied by Hatcher and Vogtmann [20].

(8) The primitivity graph $\mathcal{P}\left(F_{N}\right)$ whose vertices are conjugacy classes of primitive elements of $F_{N}$ and where two such conjugacy classes are adjacent whenever there exist a free basis $X$ of $F_{N}$ and some representatives $a_{1}, a_{2}$ of these conjugacy classes such that $a_{1}, a_{2} \in X$.

It is not hard to see that for $N \geq 3$ all of these graphs are connected and they come equipped with natural $\operatorname{Out}\left(F_{N}\right)$-actions. Moreover, with a bit of work, one can show that for a given $N \geq 3$ there are at most two substantially distinct objects among the above graphs.

More specifically, the graphs $\mathcal{F}\left(F_{N}\right)$ and $\mathcal{S}\left(F_{N}\right)$ are quasi-isometric (this is almost immediate from the definitions). Moreover, for $N \geq 3$ the graphs $\mathcal{F}^{*}\left(F_{N}\right), \mathcal{S}^{*}\left(F_{N}\right)$, $\mathcal{Z}\left(F_{N}\right), \mathcal{J}\left(F_{N}\right), \mathcal{D}\left(F_{N}\right)$ and $\mathcal{P}\left(F_{N}\right)$ are all quasi-isometric. Also, the full subgraph of $\mathcal{Z}\left(F_{N}\right)$ induced by all the vertices coming from $\mathcal{S}\left(F_{N}\right)$ together with conjugacy classes of primitive elements of $F_{N}$ can be shown to be a cobounded subset of $\mathcal{Z}\left(F_{N}\right)$ and is thus quasi-isometric to $\mathcal{Z}\left(F_{N}\right)$.

Note that there are natural $\operatorname{Out}\left(F_{N}\right)$-equivariant Lipschitz maps $j: \mathcal{F}\left(F_{N}\right) \rightarrow \mathcal{F}^{*}\left(F_{N}\right)$ and $q: \mathcal{F}^{*}\left(F_{N}\right) \rightarrow \mathcal{I}_{0}\left(F_{N}\right)$. The map $j$ is the identity map on the vertex set of $\mathcal{F}\left(F_{N}\right)$ (recall that by definition the vertex sets of $\mathcal{F}\left(F_{N}\right)$ and $\mathcal{F}^{*}\left(F_{N}\right)$ are equal). The map $q$ sends a vertex $T$ of $\mathcal{F}^{*}\left(F_{N}\right)$ to a vertex $[T]$ of $\mathcal{I}_{0}\left(F_{N}\right)$.

Note that if $T_{1}$ and $T_{2}$ are adjacent in $\mathcal{F}\left(F_{N}\right)$ and the tree $T$ corresponds to their common refinement, then for any nontrivial elliptic element $a$ for $T$ we have $\|a\|_{T}=$ $\|a\|_{T_{1}}=\|a\|_{T_{2}}=0$. Hence $T_{1}$ and $T_{2}$ are adjacent in $\mathcal{F}^{*}\left(F_{N}\right)$ and therefore the map $j$ is 1 -Lipschitz.

Similarly, suppose that two vertices $T_{1}$ and $T_{2}$ of $\mathcal{F}^{*}\left(F_{N}\right)$ are adjacent in $\mathcal{F}^{*}\left(F_{N}\right)$. Hence there exists a nontrivial element $a \in F_{N}$ such that $\|a\|_{T_{1}}=\|a\|_{T_{2}}=0$. Hence by the properties of the intersection form $\left\langle T_{i}, \eta_{a}\right\rangle=\|a\|_{T_{i}}=0$ for $i=1,2$. Hence both $\left[T_{1}\right]$ and $\left[T_{2}\right]$ are adjacent to $\left[\eta_{a}\right]$ in $\mathcal{I}\left(F_{N}\right)$ which implies that the map $q$ is 2-Lipschitz.

It appears (although we do not know how to prove this) that the map $j$, although Lipschitz, is not a quasi-isometry, and that the fibers of $j$ have infinite diameter as subsets of $\mathcal{F}^{*}\left(F_{N}\right)$.

Since the maps $q, j$ and $q \circ j$ are Lipschitz, Corollary 8.3 immediately implies analogous statements for the above graphs: 
Corollary 8.5 Let $N \geq 3$ and let $\varphi \in \operatorname{Out}\left(F_{N}\right)$ be an atoroidal iwip. Let $\mathcal{Y}$ be one of the graphs $\mathcal{F}\left(F_{N}\right), \mathcal{F}^{*}\left(F_{N}\right)$. Then for any vertex $x$ of $\mathcal{Y}$ we have

$$
\lim _{n \rightarrow \infty} d_{\mathcal{Y}}\left(\varphi^{n} x, x\right)=\infty .
$$

In particular, $\operatorname{diam}(\mathcal{Y})=\infty$.

The above corollary, together with Corollary 8.3, implies Theorem B from the introduction.

\section{References}

[1] J Behrstock, M Bestvina, M Clay, Growth of intersection numbers for free group automorphisms arXiv:0806.4975

[2] M Bestvina, M Feighn, Outer limits Available at http:// andromeda.rutgers.edu/ feighn/papers/outer.pdf

[3] M Bestvina, M Feighn, The topology at infinity of Out $\left(F_{n}\right)$, Invent. Math. 140 (2000) 651-692 MR1760754

[4] M Bestvina, M Feighn, M Handel, A hyperbolic Out $\left(F_{n}\right)$-complex arXiv: 0808.3730

[5] M Bestvina, M Handel, Train tracks and automorphisms of free groups, Ann. of Math. (2) 135 (1992) 1-51 MR1147956

[6] F Bonahon, Bouts des variétés hyperboliques de dimension 3, Ann. of Math. (2) 124 (1986) 71-158 MR847953

[7] F Bonahon, The geometry of Teichmüller space via geodesic currents, Invent. Math. 92 (1988) 139-162 MR931208

[8] F Bonahon, Geodesic currents on negatively curved groups, from: "Arboreal group theory (Berkeley, CA, 1988)", (R C Alperin, editor), Math. Sci. Res. Inst. Publ. 19, Springer, New York (1991) 143-168 MR1105332

[9] M M Cohen, M Lustig, Very small group actions on $\mathbf{R}$-trees and Dehn twist automorphisms, Topology 34 (1995) 575-617 MR1341810

[10] T Coulbois, A Hilion, M Lustig, $\mathbb{R}$-trees and laminations for free groups. I. Algebraic laminations, J. Lond. Math. Soc. (2) 78 (2008) 723-736 MR2456901

[11] T Coulbois, A Hilion, M Lustig, $\mathbb{R}$-trees and laminations for free groups. II. The dual lamination of an $\mathbb{R}$-tree, J. Lond. Math. Soc. (2) 78 (2008) 737-754 MR2456902

[12] T Coulbois, A Hilion, M Lustig, $\mathbb{R}$-trees and laminations for free groups. III. Currents and dual $\mathbb{R}$-tree metrics, J. Lond. Math. Soc. (2) 78 (2008) 755-766 MR2456903 
[13] M Culler, K Vogtmann, Moduli of graphs and automorphisms of free groups, Invent. Math. 84 (1986) 91-119 MR830040

[14] S Francaviglia, Geodesic currents and length compactness for automorphisms of free groups, Trans. Amer. Math. Soc. 361 (2009) 161-176 MR2439402

[15] D Gaboriau, A Jaeger, G Levitt, M Lustig, An index for counting fixed points of automorphisms of free groups, Duke Math. J. 93 (1998) 425-452 MR1626723

[16] E Ghys, P de la Harpe (editors), Sur les groupes hyperboliques d'après Mikhael Gromov, Progress in Math. 83, Birkhäuser, Boston (1990) MR1086648 Papers from the Swiss Seminar on Hyperbolic Groups held in Bern, 1988

[17] V Guirardel, Dynamics of $\operatorname{Out}\left(F_{n}\right)$ on the boundary of outer space, Ann. Sci. École Norm. Sup. (4) 33 (2000) 433-465 MR1832819

[18] V Guirardel, Cour et nombre d'intersection pour les actions de groupes sur les arbres, Ann. Sci. École Norm. Sup. (4) 38 (2005) 847-888 MR2216833

[19] U Hamenstädt, Subgroups of $\operatorname{Out}\left(F_{n}\right)$, Lecture at the workshop "Discrete groups and geometric structures", Kortrijk, Belgium (2008)

[20] A Hatcher, K Vogtmann, The complex of free factors of a free group, Quart. J. Math. Oxford Ser. (2) 49 (1998) 459-468 MR1660045

[21] J Hempel, 3-manifolds as viewed from the curve complex, Topology 40 (2001) 631657 MR1838999

[22] V Kaimanovich, I Kapovich, P Schupp, The subadditive ergodic theorem and generic stretching factors for free group automorphisms, Israel J. Math. 157 (2007) 1-46 MR2342439

[23] I Kapovich, The frequency space of a free group, Internat. J. Algebra Comput. 15 (2005) 939-969 MR2197815

[24] I Kapovich, Currents on free groups, from: "Topological and asymptotic aspects of group theory”, (R Grigorchuk, M Mihalik, M Sapir, Z S̆uniḱ, editors), Contemp. Math. 394, Amer. Math. Soc. (2006) 149-176 MR2216713

[25] I Kapovich, Clusters, currents, and Whitehead's algorithm, Experiment. Math. 16 (2007) 67-76 MR2312978

[26] I Kapovich, M Lustig, Domains of proper discontinuity on the boundary of Outer space arXiv:0902.4263

[27] I Kapovich, M Lustig, Intersection form, laminations and currents on free groups, to appear in Geom. Funct. Anal. arXiv:math.GR/0711.4337

[28] I Kapovich, M Lustig, Ping-pong and Outer space arXiv:0902.4017

[29] I Kapovich, M Lustig, The actions of $\operatorname{Out}\left(F_{k}\right)$ on the boundary of outer space and on the space of currents: minimal sets and equivariant incompatibility, Ergodic Theory Dynam. Systems 27 (2007) 827-847 MR2322181 
[30] I Kapovich, T Nagnibeda, The Patterson-Sullivan embedding and minimal volume entropy for outer space, Geom. Funct. Anal. 17 (2007) 1201-1236 MR2373015

[31] G Levitt, M Lustig, Irreducible automorphisms of $F_{n}$ have north-south dynamics on compactified outer space, J. Inst. Math. Jussieu 2 (2003) 59-72 MR1955207

[32] M Lustig, A generalized intersection form for free groups, Preprint (2004)

[33] M Lustig, Conjugacy and centralizers for iwip automorphisms of free groups, from: "Geometric group theory", (G N Arzhantseva, L Bartholdi, J Burillo, E Ventura, editors), Trends Math., Birkhäuser, Basel (2007) 197-224 MR2395795

[34] R Martin, Nonuniquely ergodic foliations of thin type, measured currents and automorphisms of free groups, PhD thesis, UCLA (1995)

[35] H A Masur, Y N Minsky, Geometry of the complex of curves. I. Hyperbolicity, Invent. Math. 138 (1999) 103-149 MR1714338

[36] F Paulin, The Gromov topology on $\mathbf{R}$-trees, Topology Appl. 32 (1989) 197-221 MR1007101

[37] W P Thurston, The geometry and topology of three-manifolds, Princeton Univ. Math. Dept. Lecture Notes (1979) Available at http://msri.org/publications/books/ gt $3 \mathrm{~m} /$

[38] H Zieschang, E Vogt, H-D Coldewey, Flächen und ebene diskontinuierliche Gruppen, Lecture Notes in Math. 122, Springer, Berlin (1970) MR0262378

Department of Mathematics, University of Illinois at Urbana-Champaign 1409 West Green Street, Urbana, IL 61801, USA

Mathématiques (LATP), Université Paul Cézanne - Aix Marseille III ave Escadrille Normandie-Niémen, 13397 Marseille 20, France

kapovich@math.uiuc.edu, Martin.Lustig@univ-cezanne.fr http://www . math.uiuc . edu/ kapovich/

Proposed: Walter Neumann

Seconded: Martin Bridson, Benson Farb
Received: 26 August 2008 Accepted: 6 November 2008 